\title{
LAPLACIAN COMPARISON THEOREM ON RIEMANNIAN MANIFOLDS WITH MODIFIED $m$-BAKRY-EMERY RICCI LOWER BOUNDS FOR $m \leq 1$
}

\author{
KAZUHIRO KUWAE* AND TOSHIKI SHUKURI
}

\begin{abstract}
In this paper, we prove a Laplacian comparison theorem for non-symmetric diffusion operator on complete smooth $n$-dimensional Riemannian manifold having a lower bound of modified $m$-Bakry-Émery Ricci tensor under $m \leq 1$ in terms of vector fields. As consequences, we give the optimal conditions for modified $m$-Bakry-Émery Ricci tensor under $m \leq 1$ such that the (weighted) Myers' theorem, Bishop-Gromov volume comparison theorem, Ambrose-Myers' theorem, Cheng's maximal diameter theorem, and the Cheeger-Gromoll type splitting theorem hold. Some of these results were well-studied for $m$-Bakry-Émery Ricci curvature under $m \geq n([19,21,27$, 33]) or $m=1$ (34, 35) if the vector field is a gradient type. When $m<1$, our results are new in the literature.
\end{abstract}

\section{IntRoduCtion}

1.1. Modified Bakry-Émery Ricci curvatures. Let $(M, g)$ be an $n$-dimensional smooth complete Riemannian manifold with its volume measure $\mathfrak{m}:=\operatorname{vol}_{g}$ and $V$ a $C^{1}$ vector field. Throughout this paper, we assume that the manifold $M$ has no boundary and is connected. We consider a diffusion operator $\Delta_{V}:=\Delta-\langle V, \nabla \cdot\rangle$. In [31, 32, 34], $\Delta_{V}$ is called the $V$-Laplacian on $(M, g)$.

For any constant $m \in]-\infty,+\infty]$, we introduce the symmetric 2-tensor

$$
\operatorname{Ric}_{m, n}\left(\Delta_{V}\right)(x)=\operatorname{Ric}(x)+\frac{1}{2} \mathcal{L}_{V} g(x)-\frac{V^{*}(x) \otimes V^{*}(x)}{m-n}, \quad x \in M,
$$

and call it the modified m-Bakry-Émery Ricci tensor of the diffusion operator $\Delta_{V}$. Here $\mathcal{L}_{V} g(X, Y):=\left\langle\nabla_{X} V, Y\right\rangle+\left\langle\nabla_{Y} V, X\right\rangle$ is the Lie derivative of $g$ with respect to $V$ and $V^{*}$ is the dual 1-form of $V$ coming from $g$.

For any $m \in]-\infty,+\infty]$ and a continuous function $K: M \rightarrow \mathbb{R}$, we call $(M, g, V)$ or $L$ satisfies the $\mathrm{CD}(K, m)$-condition if

$$
\operatorname{Ric}_{m, n}\left(\Delta_{V}\right)(x) \geq K(x) \quad \text { for all } \quad x \in M .
$$

2020 Mathematics Subject Classification. Primary 53C20; Secondary 53C21, 53C22, 53C23, 53C24, $58 \mathrm{~J} 60$.

Key words and phrases. modified $m$-Bakry-Émery Ricci curvature, Laplacian comparison theorem, weighted Myers' theorem, Bishop-Gromov volume comparison theorem, Ambrose-Myers' theorem, Cheng's maximal diameter theorem, Cheeger-Gromoll splitting theorem.

* Supported in part by JSPS Grant-in-Aid for Scientific Research (KAKENHI) 17H02846 and by fund (No.:185001) from the Central Research Institute of Fukuoka University. 
When $m=n$, we always assume that $V$ vanishes so that $\operatorname{Ric}_{n, n}\left(\Delta_{V}\right)=$ Ric. When $m \geq n, m$ is regarded as an upper bound for the dimension of the diffusion operator $\Delta_{V}$. Throughout this paper, we focus on the case $m \leq 1$ and assume $n>1$ if $m=1$ and $V$ does not vanish (i.e., $V \equiv 0$ and $\Delta_{V}=\Delta$ if $m=n=1$ ). Consequently, for $m \leq 1$, we always assume $n>m$ provided $V$ does not vanish. Note that, for $m \leq 1, N \in[n,+\infty[$, and for any $x \in M$, we have

$$
\operatorname{Ric}_{1, n}\left(\Delta_{V}\right)(x) \geq \operatorname{Ric}_{m, n}\left(\Delta_{V}\right)(x) \geq \operatorname{Ric}_{\infty, n}\left(\Delta_{V}\right)(x) \geq \operatorname{Ric}_{N, n}\left(\Delta_{V}\right)(x) .
$$

If we only consider the case that the lower bounds of the above Ricci tensor are constant, $\operatorname{Ric}_{1, n}\left(\Delta_{V}\right) \geq$ const. is the weakest one among them. But if we consider the case that the lower bound of Ricci curvature depends on the parameter $m$ like (2.11) below, the similar condition is no longer the weakest one.

In the literature, there have been intensive works on the study of geometry and analysis of weighted complete Riemannian manifolds with the $\mathrm{CD}(K, m)$-condition for $m \geq n$ and $K \in \mathbb{R}$ (or $K \in C(M, \mathbb{R})$ ) in the case $V=\nabla \phi$ for $\phi \in C^{2}(M)$. See [2, 3, 4, 5, 6. 17, 8, 12, 13, 19, 20, 17, 18, 21, 27, 33], and reference therein. During recent years, there are already several papers on the study of weighted Riemannian manifolds with $m$ Bakry-Émery Ricci curvature for $m<0$ or $m<1$ with $V=\nabla \phi$ for a $C^{2}$-function $\phi$. For $V=\nabla \phi$, we write $L:=\Delta_{\nabla \phi}$ in this introduction. In [25], Ohta and Takatsu proved the $K$ displacement convexity of the Rényi type entropy under the $m$-Bakry-Émery Ricci tensor condition $\operatorname{Ric}_{m, n}(L) \geq K$, i.e., the $\operatorname{CD}(K, m)$-condition, for $\left.m \in\right]-\infty, 0[\cup[n,+\infty[$ and $K \in \mathbb{R}$. After that, Ohta [24] and Kolesnikov-Milman [15] simultaneously treated the case $m<0$. Ohta [24] extended the Bochner inequality, eigenvalue estimates, and the BrunnMinkowski inequality under the lower bound for $\operatorname{Ric}_{m, n}(L)$ with $m<0$. KolesnikovMilman [15] also proved the Poincaré and the Brunn-Minkowski inequalities for manifolds with boundary under the lower bound for $\operatorname{Ric}_{m, n}(L)$ with $m<0$. In [24, Theorem 4.10], Ohta also proved that the lower bound of $\operatorname{Ric}_{m, n}(L)(x)$ with $m<0$ is equivalent to the curvature dimension condition in terms of mass transport theory as defined by Lott-Villani [22] and Sturm [29, 30]. In [34], Wylie proved a warped product version of Cheeger-Gromoll splitting theorem under the $\mathrm{CD}(0,1)$-condition. He also proved an isometric product version of Cheeger-Gromoll splitting under $\operatorname{CD}(0, m)$-condition with $m<1$ and $(V, 1)$-completeness condition. In [35], W. Wylie and D. Yeroshkin proved a Laplacian comparison theorem, a Bishop-Gromov volume comparison theorem, Myers' theorem and Cheng's maximal diameter theorem on manifolds with $m$-Bakry-Émery Ricci curvature condition for $m=1$ with $V=\nabla \phi$ for a $C^{2}$-function $\phi$. Recently, Milman [23] extended the Heintze-Karcher Theorem, isoperimetric inequality, and functional inequalities under the lower bound for $\operatorname{Ric}_{m, n}(L)(x)$ with $m<1$. In [16], the first named author and X.-D. Li established the Laplacian comparison theorem on weighted complete Riemannian manifolds with the $\operatorname{CD}(K, m)$-condition with $m \leq 1$ for $V=\nabla \phi$ with 
$\phi \in C^{2}(M)$, and obtained (weighted) Myers' theorem, Bishop-Gromov volume comparison theorem, Ambrose-Myers' theorem, Cheeger-Gromoll type splitting theorem, stochastic completeness and Feller property of $L$-diffusion process under optimal conditions on the $m$-Bakry-Émery Ricci tensor for $m \leq 1$ over the weighted complete Riemannian manifolds.

It is important to know whether one can establish the Laplacian comparison theorem on such Riemannian manifolds with the $\operatorname{CD}(K, m)$-condition for $m \leq 1$ and $K \in \mathbb{R}$ for general $C^{1}$-vector field $V$. In this paper, we prove such comparison theorem for $K$ being a continuous function depending on a re-parametrized distance function on $M$. As consequences, we give the optimal conditions on the modified $m$-Bakry-Émery Ricci tensor for $m \leq 1$ so that (weighted) Myers' theorem, Bishop-Gromov volume comparison theorem, Ambrose-Myers' theorem, Cheng's maximal diameter theorem, and the CheegerGromoll type splitting theorem hold on weighted complete Riemannian manifolds. These geometric results are complete extensions of the case for $V=\nabla \phi$ proved in the first part of [16]. When $m<1$, our results are new in the literature.

Acknowledgment. The authors would thank to Dr. Yohei Sakurai for his significant comments to the draft of this paper. They also would like to thank to the anonymous referee. His/Her comments help to improve the quality of this paper very much.

\section{MAIN RESULT}

Let $V$ be a $C^{1}$-vector field on a Riemannian manifold $(M, g)$. Since there may be no function $\phi$ satisfying $V=\nabla \phi$ in general, we can still make sense of bounds by integrating $V$ along geodesics. Define

$$
V_{\gamma}(r):=\int_{0}^{r}\left\langle V_{\gamma_{s}}, \dot{\gamma}_{s}\right\rangle \mathrm{d} s
$$

for a unit speed geodesic $\gamma:[0, T[\rightarrow M$, and

$$
\phi_{V}(x):=\inf \left\{\begin{array}{l|l}
\int_{0}^{r_{p}(x)}\left\langle V_{\gamma_{s}}, \dot{\gamma}_{s}\right\rangle \mathrm{d} s & \begin{array}{l}
\gamma: \text { unit speed geodesic } \\
\gamma_{0}=p, \gamma\left(r_{p}(x)\right)=x
\end{array}
\end{array}\right\} .
$$

Note that $V_{\gamma}$ depends on the choice of unit speed geodesic $\gamma$, and $\phi_{V}(x)$ depends on $p$ with $\phi_{V}(p)=0$ and it is well-defined for $x \in M$. It is easy to see that $\phi_{V}(x)=\int_{0}^{r_{p}(x)} V r_{p}\left(\gamma_{s}\right) \mathrm{d} s$ under $x \notin \operatorname{Cut}(p)$, where $\gamma$ is the unique unit speed geodesic with $\gamma_{0}=p$ and $\gamma\left(r_{p}(x)\right)=$ $x$. Hence $\phi_{V}$ is a continuous function on $(\operatorname{Cut}(p) \cup\{p\})^{c}$. Consequently, $\phi_{V}$ is an mmeasurable function. Moreover, for $x \notin \operatorname{Cut}(p), \phi_{V}(x)=V_{\gamma}\left(r_{p}(x)\right)$ for the unique unit speed geodesic $\gamma$ with $\gamma_{0}=p$ and $\gamma\left(r_{p}(x)\right)=x$. Hence $\phi_{V}\left(\gamma_{t}\right)=V_{\gamma}(t)$ for any unit speed geodesic $\gamma$ with $\gamma_{0}=p$ and $\gamma_{t} \notin \operatorname{Cut}(p)$. When $V=\nabla \phi$ is a gradient vector field for some 
$\phi \in C^{2}(M)$, then one can see

$$
V_{\gamma}(t)=\int_{0}^{t}\left\langle\nabla \phi, \dot{\gamma}_{s}\right\rangle \mathrm{d} s=\int_{0}^{t} \frac{\mathrm{d}}{\mathrm{d} s} \phi\left(\gamma_{s}\right) \mathrm{d} s=\phi\left(\gamma_{t}\right)-\phi\left(\gamma_{0}\right)
$$

Throughout this paper, we fix a point $p \in M$ and a constant $C_{p}>0$, which may depend on $p$. For $x \in M$, we define

$$
s_{p}(x):=\inf \left\{\begin{array}{l|l}
C_{p} \int_{0}^{r_{p}(x)} e^{-\frac{2 V_{\gamma}(t)}{n-m} \mathrm{~d} t} & \begin{array}{l}
\gamma: \text { unit speed geodesic } \\
\gamma_{0}=p, \gamma\left(r_{p}(x)\right)=x
\end{array}
\end{array}\right\} .
$$

If $(M, g)$ is complete, then $s_{p}(x)$ is finite and well-defined from the basic properties of Riemannian geodesics. Let $s(p, q):=s_{p}(q)$ for $p, q \in M$. If $q$ is not a cut point of $p$, then there is a unique minimal geodesic from $p$ to $q$ and $s_{p}$ is smooth in a neighborhood of $q$ as can be computed by pulling the function back by the exponential map at $p$. Note that $s(p, q) \geq 0$, it is zero if and only if $p=q$. But $s(p, q)=s(q, p)$ does not hold in general. If $V=\nabla \phi$ for some $\phi \in C^{2}(M)$ and set $C_{p}=\exp \left(-\frac{2 \phi(p)}{n-m}\right)$ for the definition of $s_{p}(x)$ with $p$ being arbitrary, then one can see that $s(p, q)=s(q, p)$ for $p, q \in M$. However, $s(p, q)$ does not necessarily satisfy the triangle inequality.

Definition 2.1. Let $(M, g)$ be an $n$-dimensional complete Riemannian manifold and $V$ a $C^{1}$-vector field. Fix $p \in M$. Then we say that $(M, g, V)$ is $(V, m)$-complete at $p$ if

$$
\varlimsup_{r \rightarrow+\infty} \inf _{L(\gamma)=r} \int_{0}^{r} e^{-\frac{2 V_{\gamma}(t)}{n-m}} \mathrm{~d} t=+\infty
$$

where the infimum is taken over all minimizing unit speed geodesics $\gamma$ with respect to the metric $g$ such that $\gamma_{0}=p$. We say that $(M, g, V)$ is $(V, m)$-complete if it is $(V, m)$-complete at $p$ for all $p \in M$.

REMARK 2.2. (1) If $V_{\gamma}$ is upper bounded for any unit speed geodesic $\gamma$ with $\gamma_{0}=p$, then $(M, g, V)$ is always $(V, m)$-complete at $p$ for all $m \leq 1$. In particular, if there exists a non-negative integrable function $f$ on $\left[0,+\infty\left[\right.\right.$ such that $\left\langle V, \nabla r_{p}\right\rangle_{x} \leq$ $f\left(r_{p}(x)\right)$, then $V_{\gamma}(r) \leq \int_{0}^{r} f(t) \mathrm{d} t \leq \int_{0}^{\infty} f(t) \mathrm{d} t<\infty$ so that $(M, g, V)$ is always $(V, m)$-complete at $p$ for all $m \leq 1$.

(2) If $M$ is compact, then $(M, g, V)$ is always $(V, m)$-complete for $m \leq 1$. Indeed, if so, the set $G_{r}:=\{\gamma \mid \gamma$ is a unit speed minimal geodesic, $L(\gamma)=r\}$ is an empty set for sufficiently large $r>0$. This implies (2.2) .

(3) If there exists a non-negative locally integrable function $f$ on $[0,+\infty[$ satisfying $f(t) \leq C / t$ on $[1,+\infty[$ for some $C \in] 0,(n-m) / 2]$ and $\left\langle V, \nabla r_{p}\right\rangle \leq f\left(r_{p}\right)$, then $(V, m)$-completeness at $p$ holds for all $m \leq 1$. Here we assume $n>1$ for $m=1$. 
In fact, we see for $r>1$

$$
\begin{aligned}
\inf _{L(\gamma)=r} \int_{0}^{r} e^{-\frac{2 V_{\gamma}(t)}{n-m}} \mathrm{~d} t & \geq \int_{1}^{r} e^{-\frac{2 \int_{0}^{t} f(s) \mathrm{d} s}{n-m}} \mathrm{~d} t \\
& \geq e^{-\frac{2 \int_{0}^{1} f(s) \mathrm{d} s}{n-m}} \int_{1}^{r} e^{-\frac{2 \int_{1}^{t} f(s) \mathrm{d} s}{n-m}} \mathrm{~d} t \\
& \geq e^{-\frac{2 \int_{0}^{1} f(s) \mathrm{d} s}{n-m}} \int_{1}^{r} e^{-\frac{2 C \log t}{n-m}} \mathrm{~d} t \\
& \geq e^{-\frac{2 \int_{0}^{1} f(s) \mathrm{d} s}{n-m}} \int_{1}^{r} \frac{\mathrm{d} t}{t^{\frac{2 C}{n-m}}} \rightarrow+\infty \quad \text { as } \quad r \rightarrow \infty
\end{aligned}
$$

where the infimum is taken over all minimizing unit speed geodesics $\gamma$ with $\gamma_{0}=p$.

(4) The $(V, 1)$-completeness at $p$ defined as in [34, Definition 6.2] implies the $(V, m)$ completeness at $p$ for every $m \leq 1$ provided $V_{\gamma} \geq 0$ for any unit speed geodesic $\gamma$ with $\gamma_{0}=p$. The converse also holds under $V_{\gamma} \leq 0$ for any unit speed geodesic $\gamma$ with $\gamma_{0}=p$.

Lemma 2.3. Let $(M, g)$ be an $n$-dimensional complete non-compact Riemannian manifold and $V$ a $C^{1}$-vector field. Fix $p \in M$ and suppose that $(M, g, V)$ is $(V, m)$-complete at $p$. Then, for any sequence $\left\{q_{i}\right\}$ in $M$ such that $d\left(p, q_{i}\right) \rightarrow+\infty$ as $i \rightarrow+\infty, s\left(p, q_{i}\right) \rightarrow+\infty$.

Proof. The proof is similar to that of [35, Proposition 3.4]. We omit it.

REMARK 2.4. Recall that $\phi_{V}$ depends on $p \in M$. For a fixed $p \in M$, we set $\underline{\phi}_{V}(r):=$ $\inf _{B_{r}(p)} \phi_{V}$ and $\bar{\phi}_{V}(r):=\sup _{B_{r}(p)} \phi_{V}$ for $\left.r \in\right] 0,+\infty\left[\right.$. Then $\underline{\phi}_{V}(r) \leq 0 \leq \bar{\phi}_{V}(r)$ for $r>0$ and $\lim _{r \rightarrow 0} \bar{\phi}_{V}(r)=\lim _{r \rightarrow 0} \underline{\phi}_{V}(r)=0$. If $x \notin \operatorname{Cut}(p)$, we have $s_{p}(x)=C_{p} \int_{0}^{r_{p}(x)} e^{-\frac{2 \phi_{V}\left(\gamma_{t}\right)}{n-m}} \mathrm{~d} t$ for the unique unit speed geodesic $\gamma$ with $\gamma_{0}=p$ and $\gamma\left(r_{p}(x)\right)=x$. So $\lim _{x \rightarrow p} \frac{s_{p}(x)}{r_{p}(x)}=C_{p}$. In particular,

$$
C_{p} e^{-\frac{2 \bar{\phi}_{V}\left(r_{p}(x)\right)}{n-m}} r_{p}(x) \leq s_{p}(x) \leq C_{p} e^{-\frac{2 \underline{\underline{p}}_{V}\left(r_{p}(x)\right)}{n-m}} r_{p}(x) \quad \text { for } \quad x \notin \operatorname{Cut}(p) .
$$

2.1. Laplacian Comparison. Let $\kappa:\left[0,+\infty\left[\rightarrow \mathbb{R}\right.\right.$ be a continuous function and $\mathrm{a}_{\kappa}$ the unique solution defined on the maximal interval $] 0, \delta_{\kappa}\left[\right.$ for $\left.\left.\delta_{\kappa} \in\right] 0,+\infty\right]$ of the following Riccati equation

$$
-\frac{\mathrm{d} \mathrm{a}_{\kappa}}{\mathrm{d} s}(s)=\kappa(s)+\mathrm{a}_{\kappa}(s)^{2}
$$

with the boundary conditions

$$
\lim _{s \downarrow 0} s \mathrm{a}_{\kappa}(s)=1,
$$

and

$$
\lim _{s \uparrow \delta_{\kappa}}\left(s-\delta_{\kappa}\right) \mathrm{a}_{\kappa}(s)=1
$$


under $\delta_{\kappa}<\infty$. (2.4) yields

$$
\lim _{s \downarrow 0} \mathrm{a}_{\kappa}(s)=+\infty .
$$

If $\delta_{\kappa}<\infty$, from (2.5), $\delta_{\kappa}$ is the explosion time of $\mathrm{a}_{\kappa}$ in the sense that

$$
\lim _{s \uparrow \delta_{\kappa}} \mathrm{a}_{\kappa}(s)=-\infty .
$$

Actually, $a_{\kappa}(s)=\mathfrak{s}_{\kappa}^{\prime}(s) / \mathfrak{s}_{\kappa}(s)$, where $\mathfrak{s}_{\kappa}$ is the unique solution of Jacobi equation $\mathfrak{s}_{\kappa}^{\prime \prime}(s)+$ $\kappa(s) \mathfrak{s}_{\kappa}(s)=0$ with $\mathfrak{s}_{\kappa}(0)=0, \mathfrak{s}_{\kappa}^{\prime}(0)=1$, and $\delta_{\kappa}=\inf \left\{s>0 \mid \mathfrak{s}_{\kappa}(s)=0\right\}$. We write $\mathrm{a}_{\kappa}(s)=\cot _{\kappa}(s)$. Moreover, $] 0, \delta_{\kappa}\left[\ni s \mapsto \cot _{\kappa}(s)\right.$ is decreasing (resp. strictly decreasing) provided $\kappa(s)$ is non-negative (resp. positive) for all $s \in] 0, \delta_{\kappa}[$ in view of (2.3) . If $\kappa$ is a real constant, then

$$
\mathrm{a}_{\kappa}(s)=\left\{\begin{array}{cc}
\sqrt{\kappa} \cot (\sqrt{\kappa} s) & \kappa>0 \\
1 / s & \kappa=0 \\
\sqrt{-\kappa} \operatorname{coth}(\sqrt{-\kappa} s) & \kappa<0
\end{array}\right.
$$

and $\delta_{\kappa}=\pi / \sqrt{\kappa^{+}} \leq+\infty$. Fix $\left.\left.m \in\right]-\infty, 1\right]$ and set $m_{\kappa}(s):=(n-m) \cot _{\kappa}(s)$. Then (2.3) is equivalent to

$$
-\frac{\mathrm{d} m_{\kappa}}{\mathrm{d} s}(s)=(n-m) \kappa(s)+\frac{m_{\kappa}(s)^{2}}{n-m},
$$

and (2.4) (resp. (2.5)) is equivalent to $\lim _{s \downarrow 0} s m_{\kappa}(s)=n-m\left(\operatorname{resp} . \lim _{s \uparrow \delta_{\kappa}}\left(s-\delta_{\kappa}\right) m_{\kappa}(s)=\right.$ $n-m$ under $\left.\delta_{\kappa}<\infty\right)$. In view of the uniqueness of the solution to (2.3) with (2.6), we have the scaling property $\mathrm{a}_{\kappa_{\alpha}}(s)=\frac{1}{\alpha} \mathrm{a}_{\alpha^{2} \kappa}(s / \alpha)$ for $\alpha>0$. Here $\kappa_{\alpha}(s):=\kappa(s / \alpha)$. In particular, $\mathrm{a}_{\kappa}(s)=\frac{1}{\alpha} \mathrm{a}_{\alpha^{2} \kappa}(s / \alpha)$ for $\alpha>0$ provided $\kappa$ is a constant.

Our first result is the following Laplacian comparison along unit speed geodesic on weighted complete Riemannian manifolds $(M, g, V)$ under the lower bound of modified $m$-Bakry-Émery Ricci tensor for $m \leq 1$.

Theorem 2.5 (Laplacian Comparison Theorem). Suppose that $(M, g)$ is an $n$-dimensional complete smooth Riemannian manifold and $V$ is a $C^{1}$-vector field. Fix $p \in M$. Take $R \in] 0,+\infty]$. Let $\phi_{V}$ be the function defined in (2.1). Suppose that

$$
\operatorname{Ric}_{m, n}\left(\Delta_{V}\right)_{x}\left(\nabla r_{p}, \nabla r_{p}\right) \geq(n-m) \kappa\left(s_{p}(x)\right) e^{-\frac{4 \phi_{V}(x)}{n-m}} C_{p}^{2}
$$

holds under $r_{p}(x)<R$ with $x \in(\operatorname{Cut}(p) \cup\{p\})^{c}$. Then

$$
\left(\Delta_{V} r_{p}\right)(x) \leq(n-m) \cot _{\kappa}\left(s_{p}(x)\right) e^{-\frac{2 \phi_{V}(x)}{n-m}} C_{p} .
$$

Corollary 2.6. Suppose that $(M, g)$ is an n-dimensional complete smooth Riemannian manifold and $V$ is a $C^{1}$-vector field. Fix $p \in M$ and assume $\delta_{\kappa}<\infty$. Then

$$
\lim _{s_{p}(x) \uparrow \delta_{\kappa}} \Delta_{V} r_{p}(x)=-\infty .
$$


REMARK 2.7. The sufficient condition (2.9) under $r_{p}(x)<R$ with $x \in(\operatorname{Cut}(p) \cup\{p\})^{c}$ for our Laplacian comparison theorem is weaker than the condition:

$$
\operatorname{Ric}_{m, n}\left(\Delta_{V}\right)(x) \geq(n-m) \kappa\left(s_{p}(x)\right) e^{-\frac{4 \phi_{V}(x)}{n-m}} C_{p}^{2} g_{x}
$$

under $r_{p}(x)<R$, because $\nabla r_{p}(x)$ is defined only for $x \notin \operatorname{Cut}(p) \cup\{p\}$. In particular, $\mathrm{CD}(K, m)$-condition for $K(x)=(n-m) \kappa\left(s_{p}(x)\right) e^{-\frac{4 \phi_{V}(x)}{n-m}} C_{p}^{2}$ always implies that (2.9) holds for all $x \in(\operatorname{Cut}(p) \cup\{p\})^{c}$.

REMARK 2.8. The inequality (2.10) is meaningful at $p$, because $m_{\kappa}(0+)=+\infty$ and $\Delta_{V} r_{p}(p)=\Delta r_{p}(p)=+\infty$ in view of the classical Laplacian comparison theorem for $\Delta$ under local upper sectional curvature bound (see [14, Theorem 3.4.2]). Moreover, the following inequality

$$
r_{p}(x)\left(\Delta_{V} r_{p}\right)(x) \leq(n-m) r_{p}(x) \cot _{\kappa}\left(s_{p}(x)\right) e^{-\frac{2 \phi_{V}(x)}{n-m}} C_{p}
$$

is also meaningful at $p$. Indeed, the right hand side of (2.12) has the value $n-m$ at $x=p$ by Remark 2.4 and the left hand side has the value $n-1$ at $x=p$ by the classical Laplacian comparison theorem for $\Delta$ as noted above.

REMARK 2.9. Theorem 2.5 generalizes [35, Theorem 4.4].

\subsection{Geometric consequences.}

Theorem 2.10 (Weighted Myers' Theorem). Let $(M, g)$ be an $n$-dimensional complete Riemannian manifold and a $C^{1}$-vector field $V$. Fix $p \in M$. Assume that (2.9) holds for all $x \in(\operatorname{Cut}(p) \cup\{p\})^{c}$ and $\delta_{\kappa}<\infty$. Then $s(p, q) \leq \delta_{\kappa}$ for all $q \in M$.

Corollary 2.11. Let $(M, g)$ be an n-dimensional complete Riemannian manifold and a $C^{1}$-vector field $V$. Fix $p \in M$ and $\delta_{\kappa}<\infty$. Assume that (2.9) holds for all $x \in$ $(\operatorname{Cut}(p) \cup\{p\})^{c}$ and $(M, g, V)$ is $(V, m)$-complete at $p$. Then $M$ is compact.

REMARK 2.12. (1) Theorem[2.10(resp. Corollary 2.11) generalizes [35, Theorem 2.2] (resp. [35, Corollary 2.3]).

(2) Since $V_{\gamma} \leq 0$ for any unit speed geodesic $\gamma$ with $\gamma_{0}=p$ implies the $(V, m)$ completeness at $p$, Corollary 2.11 implies the compactness of $M$ provided $\delta_{\kappa}<\infty$, (2.9) holds for $x \notin \operatorname{Cut}(p) \cup\{p\}$ and $V_{\gamma} \leq 0$ any unit speed geodesic $\gamma$ with $\gamma_{0}=p$.

Based on Theorems 2.5] and 2.10, we can deduce several geometric fruitful results. Next we will give two versions of the Bishop-Gromov type volume comparison. The first one is for $\mu_{V}(A)=\int_{A} e^{-\phi_{V}(x)} \mathfrak{m}(\mathrm{d} x)$ of metric annuli $A\left(p, r_{0}, r_{1}\right):=\left\{x \in M \mid r_{0} \leq r_{p}(x) \leq r_{1}\right\}$. 
The comparison in this case will be in terms of the quantities

$$
\begin{aligned}
\bar{\nu}_{p}\left(\kappa, r_{0}, r_{1}\right): & =\int_{r_{0}}^{r_{1}} \int_{\mathbb{S}^{n-1}} \mathfrak{s}_{\kappa}^{n-m}\left(\sup _{\eta} s_{p}(r, \eta)\right) \mathrm{d} r \mathrm{~d} \theta, \quad \bar{\nu}_{p}\left(\kappa, r_{1}\right):=\bar{\nu}_{p}\left(\kappa, 0, r_{1}\right), \\
\underline{\nu}_{p}\left(\kappa, r_{0}, r_{1}\right): & :=\int_{r_{0}}^{r_{1}} \int_{\mathbb{S}^{n-1}} \mathfrak{s}_{\kappa}^{n-m}\left(\inf _{\eta} s_{p}(r, \eta)\right) \mathrm{d} r \mathrm{~d} \theta, \quad \underline{\nu}_{p}\left(\kappa, r_{1}\right):=\underline{\nu}_{p}\left(\kappa, 0, r_{1}\right), \\
\nu_{p}\left(\kappa, r_{0}, r_{1}\right): & :=\int_{r_{0}}^{r_{1}} \int_{\mathbb{S}^{n-1}} \mathfrak{s}_{\kappa}^{n-m}\left(s_{p}(r, \theta)\right) \mathrm{d} r \mathrm{~d} \theta, \quad \nu_{p}\left(\kappa, r_{1}\right):=\nu_{p}\left(\kappa, 0, r_{1}\right)
\end{aligned}
$$

under $s_{p}\left(r_{1}, \theta\right) \leq \delta_{\kappa}$ for all $\theta \in \mathbb{S}^{n-1}$. Here

$$
s_{p}(r, \theta):=C_{p} \int_{0}^{r} e^{-\frac{2 V_{\gamma}(t)}{n-m}} \mathrm{~d} t
$$

with $\theta=\dot{\gamma}_{0}$, and $\bar{\phi}_{V}(r)$ and $\underline{\phi}_{V}(r)$ are the functions defined in Remark 2.4. If $\phi_{V}$ is rotationally symmetric around $p$, i.e., if there exists a $C^{2}$-function $\Phi_{V}$ on $[0,+\infty[$ such that $\phi_{V}(x)=\Phi_{V}\left(r_{p}(x)\right)$, then $s_{p}(r, \theta)$ is independent of $\theta \in \mathbb{S}^{n-1}$. The second one is for $\nu_{V}(A):=\int_{A} e^{-\frac{2 \phi_{V}(x)}{n-m}} \mu_{V}(\mathrm{~d} x)=\int_{A} e^{-\frac{n-m+2}{n-m} \phi_{V}(x)} \mathfrak{m}(\mathrm{d} x)$ of the sets $C\left(p, s_{0}, s_{1}\right):=\{x \in M \mid$ $\left.s_{0} \leq s_{p}(x) \leq s_{1}\right\}$ and $C_{s}(p):=C(p, 0, s)$. The set $C\left(p, s_{0}, s_{1}\right)$ also depends on $s_{p}$ and is quite different from annuli. The comparison in this case will be in terms of the quantities

$$
v\left(\kappa, s_{0}, s_{1}\right):=\int_{s_{0}}^{s_{1}} \int_{\mathbb{S}^{n-1}} \mathfrak{s}_{\kappa}^{n-m}(s) \mathrm{d} s \mathrm{~d} \theta \quad \text { and } \quad v\left(\kappa, s_{1}\right):=v\left(\kappa, 0, s_{1}\right)
$$

under $s_{1} \leq \delta_{\kappa}$. When $\left.\left.m \in\right]-\infty, 1\right]$ is an integer and $\kappa$ is a constant, (2.16) is the volume of annuli in the simply connected space form of constant curvature $\kappa$ and dimension $n-m+1$.

Theorem 2.13 (Bishop-Gromov Volume Comparison). Fix $p \in M$ and $R \in] 0,+\infty]$. Suppose that $(M, g)$ is an $n$-dimensional complete smooth Riemannian manifold and a $C^{1}$-vector field $V$. Let $\kappa:[0,+\infty[\rightarrow \mathbb{R}$ be a continuous function. Assume that (2.9) holds for $r_{p}(x)<R$ with $x \in(\operatorname{Cut}(p) \cup\{p\})^{c}$. Then we have the following:

(1) Suppose that $0 \leq r_{0}<r_{a} \leq r_{1}$ and $0 \leq r_{0} \leq r_{b}<r_{1}$. Then

$$
\frac{\mu_{V}\left(A\left(p, r_{b}, r_{1}\right)\right)}{\mu_{V}\left(A\left(p, r_{0}, r_{a}\right)\right)} \leq \frac{\bar{\nu}_{p}\left(\kappa, r_{b}, r_{1}\right)}{\underline{\nu}_{p}\left(\kappa, r_{0}, r_{a}\right)}
$$

holds for $r_{1}<R$. Assume further that $\phi_{V}$ is rotationally symmetric around $p$. Then

$$
\frac{\mu_{V}\left(A\left(p, r_{b}, r_{1}\right)\right)}{\mu_{V}\left(A\left(p, r_{0}, r_{a}\right)\right)} \leq \frac{\nu_{p}\left(\kappa, r_{b}, r_{1}\right)}{\nu_{p}\left(\kappa, r_{0}, r_{a}\right)}
$$

holds for $r_{1}<R$, in particular, the function

$$
] 0, R\left[\ni r \mapsto \frac{\mu_{V}\left(B_{r}(p)\right)}{\nu_{p}(\kappa, r)}\right.
$$

is non-increasing. 
(2) Suppose that $0 \leq s_{0}<s_{a} \leq s_{1}$ and $0 \leq s_{0} \leq s_{b}<s_{1}$. Then

$$
\frac{\nu_{V}\left(C\left(p, s_{b}, s_{1}\right)\right)}{\nu_{V}\left(C\left(p, s_{0}, s_{a}\right)\right)} \leq \frac{v\left(\kappa, s_{b}, s_{1}\right)}{v\left(\kappa, s_{0}, s_{a}\right)}
$$

holds for $s_{1}<S$. In particular, the function

$$
] 0, S\left[\ni s \mapsto \frac{\nu_{V}\left(C_{s}(p)\right)}{v(\kappa, s)}\right.
$$

is non-increasing. Here $S=\inf _{\theta \in \mathbb{S}^{n-1}} s_{p}(R, \theta)$.

REMARK 2.14. (2.19) (resp. (2.21)) may not be bounded as $r \rightarrow 0$ (resp. $s \rightarrow 0$ ) unless $m=1$. Note that the Bishop type inequality holds for $m=1$ (see [35, Corollary 4.6]).

Corollary 2.15. Fix $p \in M$ and $R \in] 0,+\infty]$. Suppose that $(M, g)$ is an $n$-dimensional complete smooth Riemannian manifold and $V$ is a $C^{1}$-vector field. Assume that

$$
\operatorname{Ric}_{m, n}\left(\Delta_{V}\right)_{x}\left(\nabla r_{p}, \nabla r_{p}\right) \geq 0 \quad \text { for } \quad r_{p}(x)<R \quad \text { with } \quad x \notin \operatorname{Cut}(p) \cup\{p\} .
$$

Then

$$
\frac{\mu_{V}\left(B_{r_{2}}(p)\right)}{\mu_{V}\left(B_{r_{1}}(p)\right)} \leq e^{2\left(\bar{\phi}_{V}\left(r_{1}\right)-\underline{\phi}_{V}\left(r_{2}\right)\right)}\left(\frac{r_{2}}{r_{1}}\right)^{n-m+1} \quad \text { for all } \quad 0<r_{1}<r_{2}<R
$$

holds.

Theorem 2.16 (Ambrose-Myers' Theorem). Let $(M, g)$ be an $n$-dimensional complete Riemannian manifold and $V$ a $C^{1}$-vector field. Fix $p \in M$. Assume that $(M, g, V)$ is $(V, m)$-complete at $p$. Suppose that for every unit speed (local minimizing) geodesic $\gamma$ with $\gamma_{0}=p$, we have

$$
\int_{0}^{\infty} e^{\frac{2 V_{\gamma}(t)}{n-m}} \operatorname{Ric}_{m, n}\left(\Delta_{V}\right)\left(\dot{\gamma}_{t}, \dot{\gamma}_{t}\right) \mathrm{d} t=+\infty
$$

Then $M$ is compact.

Corollary 2.17. Let $(M, g)$ be an $n$-dimensional complete Riemannian manifold and $V$ a $C^{1}$-vector field. Fix $p \in M$. Assume $\operatorname{Ric}_{m, n}\left(\Delta_{V}\right) \geq 0$ on $M$. Suppose that there exists a non-negative measurable function $f$ on $\left[0,+\infty\right.$ [ satisfying $\int_{0}^{\infty} f(s) \mathrm{d} s<+\infty$ and $\left\langle V, \nabla r_{p}\right\rangle \geq-f\left(r_{p}\right)$, and for every unit speed (local minimizing) geodesic $\gamma$ with $\gamma_{0}=p$, we have

$$
\int_{0}^{\infty} \operatorname{Ric}_{m, n}\left(\Delta_{V}\right)\left(\dot{\gamma}_{t}, \dot{\gamma}_{t}\right) \mathrm{d} t=+\infty
$$

Then $M$ is compact.

Corollary 2.18. Let $(M, g)$ be an $n$-dimensional complete Riemannian manifold and $V$ a $C^{1}$-vector field. Fix $p \in M$ and a constant $\kappa>0$. Assume that $(M, g, V)$ is $(V, m)$ complete at $p$. Suppose that for every unit speed (local minimizing) geodesic $\gamma$ with $\gamma_{0}=p$, 
we have

$$
\operatorname{Ric}_{m, n}\left(\Delta_{V}\right)\left(\dot{\gamma}_{t}, \dot{\gamma}_{t}\right) \geq(n-m) \kappa e^{-\frac{4 V_{\gamma}(t)}{n-m}} C_{p}^{2}
$$

Then $M$ is compact.

REMARK 2.19. (1) Theorem 2.16] is a version of Ambrose's Theorem ([1]). Here Ambrose's Theorem states that if for any (local minimizing) geodesic $\gamma$ emanating from a point $p \in M$,

$$
\int_{0}^{\infty} \operatorname{Ric}\left(\dot{\gamma}_{t}, \dot{\gamma}_{t}\right) \mathrm{d} t=+\infty
$$

then $M$ is compact. Cavalcante-Oliveira-Santos [10] also proved the following different version of Ambrose's Theorem (see [10, Theorem 2.1]): Suppose that every (local minimizing) geodesic $\gamma$ emanating from $p$ satisfies

$$
\int_{0}^{\infty} \operatorname{Ric}_{m, n}\left(\Delta_{\nabla \phi}\right)\left(\dot{\gamma}_{t}, \dot{\gamma}_{t}\right) \mathrm{d} t=+\infty
$$

under $m>n$ for $\phi \in C^{2}(M)$. Then $M$ is compact. Tadano [31, Theorem 14] extends [10, Theorem 2.1] for $\Delta_{V}$ with modified $m$-Bakry-Émery Ricci tensor $\operatorname{Ric}_{m, n}\left(\Delta_{V}\right)$ under $m>n$. Our Theorem 2.16 is different from the above mentioned results. Tadano [32, Theorem 25] also proves a version of Ambrose's Theorem for $\Delta_{V}$ with modified 1-Bakry-Émery Ricci tensor $\operatorname{Ric}_{1, n}\left(\Delta_{V}\right)$ under the condition $\operatorname{Ric}_{1, n}\left(\Delta_{V}\right)>0$ and $|V| \leq k e^{-\ell r_{p}}$ for some $k \geq 0, \ell>0$. So our condition in Corollary 2.17 is milder than one in [32, Theorem 25].

In the following theorem and its corollary, we assume $V=\nabla \phi$ for some $\phi \in C^{2}(M)$ and set $C_{p}=\exp \left(-\frac{2 \phi(p)}{n-m}\right)$ for the definition of $s_{p}(x)$ with $p$ being an arbitrary point. As noted before, $s(p, q)$ is symmetric for any $p, q \in M$. Let $h=e^{-\frac{4 \phi}{n-m}} g$ be the conformal change of the metric $g$. Then $s(p, q)$ is the smallest length in the $h$ metric of a minimal geodesic between $p$ and $q$ in the $g$ metric. As such, $d^{h}(p, q) \leq s(p, q)$ for any $q \in M$. So Theorem 2.10 tells us that the diameter of the metric $h$ is less than or equal to $\delta_{\kappa}$. For this conformal diameter estimate we also obtain the following rigidity characterization.

Theorem 2.20 (Cheng's Maximal Diameter Theorem). Suppose that $(M, g), n>1$, is a complete Riemannian manifold and $\phi \in C^{2}(M)$. Fix $p, q \in M$. Assume that $\delta_{\kappa}<$ $\infty, \kappa$ is positive on $] 0, \delta_{\kappa}\left[, \kappa(s)=\kappa\left(\delta_{\kappa}-s\right)\right.$ for all $s \in\left[0, \delta_{\kappa}\right]$, and (2.9) holds for all $x \in(\operatorname{Cut}(p) \cup\{p\})^{c}$. We further assume that (2.9) by replacing $p$ with $q$ holds for all $x \in(\operatorname{Cut}(q) \cup\{q\})^{c}$. If $d^{h}(p, q)=\delta_{\kappa}$, then $m=1, \phi$ is rotationally symmetric around $p$, i.e., $\phi$ is a function depending only on radial $r$, and $g$ is a warped product metric of the form

$$
g=\mathrm{d} r^{2}+e^{\frac{2 \phi(r)+2 \phi(0)}{n-1}} \mathfrak{s}_{\kappa}^{2}(s(r)) g_{\mathbb{S}^{n-1}}, \quad 0 \leq r \leq d(p, q),
$$

where $s(r)=\int_{0}^{r} e^{-\frac{2 \phi(t)}{n-1}} \mathrm{~d} t$ and $s(d(p, q))=\delta_{\kappa}$. 
Corollary 2.21. Suppose that $(M, g), n>1$, is a complete Riemannian manifold and $\phi \in C^{2}(M)$. Fix $p, q \in M$. Assume that $\kappa$ is a positive constant and (2.9) holds for all $x \in(\operatorname{Cut}(p) \cup\{p\})^{c}$. We further assume that (2.9) by replacing $p$ with $q$ holds for all $x \in(\operatorname{Cut}(q) \cup\{q\})^{c}$. If $d^{h}(p, q)=\pi / \sqrt{\kappa}$, then $m=1, \phi$ is rotationally symmetric around $p$, i.e., $\phi$ is a function depending only on radial $r$, and $g$ is a warped product metric of the form

$$
g=\mathrm{d} r^{2}+e^{\frac{2 \phi(r)+2 \phi(0)}{n-1}} \cdot \frac{\sin ^{2}(\sqrt{\kappa}(s(r)))}{\kappa} g_{\mathbb{S}^{n-1}}, \quad 0 \leq r \leq d(p, q),
$$

where $s(r)=\int_{0}^{r} e^{-\frac{2 \phi(t)}{n-1}} \mathrm{~d} t$ and $s(d(p, q))=\pi / \sqrt{\kappa}$.

Theorem 2.22 (Cheeger-Gromoll Splitting Theorem). Let $(M, g)$ be an $n$-dimensional non-compact complete Riemannian manifold and $V$ a $C^{1}$-vector field. Suppose that $(M, g, V)$ is $(V, m)$-complete and $M$ contains a line. Then under $\operatorname{CD}(0, m)$-condition with $m<1$, $M$ is isometric to $\mathbb{R} \times N$ and $V$ depends only on $N$.

Corollary 2.23. Let $(M, g)$ be an n-dimensional non-compact complete Riemannian manifold and $C^{1}$-vector field $V$. Suppose that $V_{\gamma} \leq 0$ for any unit speed geodesic $\gamma$ and $M$ contains a line. Then under $\mathrm{CD}(0, m)$-condition with $m<1$, we have that $M$ is isometric to $\mathbb{R} \times N$ and $V$ depends only on $N$.

Proof. If $V_{\gamma} \leq 0$ for any unit speed geodesic $\gamma$, then $(M, g, V)$ is $(V, m)$-complete for all $m \leq 1$. So the assertion easily follows Theorem 2.22 .

REMARK 2.24. Theorem 2.22 partially extends [34, Corollary 6.7] for a restricted case, where $\mathrm{CD}(0, m)$-condition for $m<1$ and $(V, 1)$-completeness of $(M, g, V)$ are assumed for the isometric splitting $M=\mathbb{R} \times N$. Note that the $(V, m)$-completeness does not necessarily mean the $(V, 1)$-completeness, and it is weaker than $(V, 1)$-completeness if $V_{\gamma} \geq 0$ for any unit speed geodesic $\gamma$.

\section{Proof of Theorem 2.5}

Recall the $V$-Laplacian $\Delta_{V} u:=\Delta u-\langle V, \nabla u\rangle$. Letting $\lambda(r, \theta)=C_{p}^{-1} e^{\frac{2 V_{\gamma}(r)}{n-m}} \Delta_{V} r_{p}(r, \theta)$, we find that $\lambda$ satisfies the Riccati differential inequality in terms of the parameter $s$.

LEMma 3.1. Let $\gamma$ be a unit speed minimal geodesic with $\gamma_{0}=p$ and $\dot{\gamma}_{0}=\theta$. Let $s$ be the parameter $\mathrm{d} s=C_{p} e^{-\frac{2 V_{\gamma}(r)}{n-m}} \mathrm{~d} r$. Then

$$
\frac{\mathrm{d} \lambda}{\mathrm{d} s} \leq-\frac{\lambda^{2}}{n-m}-C_{p}^{-2} e^{\frac{4 V_{\gamma}(r)}{n-m}} \operatorname{Ric}_{m, n}\left(\Delta_{V}\right)\left(\dot{\gamma}_{r}, \dot{\gamma}_{r}\right)
$$

in particular,

$$
\frac{\mathrm{d} \lambda}{\mathrm{d} r} \leq-C_{p} e^{-\frac{2 V_{\gamma}(r)}{n-m}} \frac{\lambda^{2}}{n-m}-C_{p}^{-1} e^{\frac{2 V_{\gamma}(r)}{n-m}} \operatorname{Ric}_{m, n}\left(\Delta_{V}\right)\left(\dot{\gamma}_{r}, \dot{\gamma}_{r}\right)
$$

holds for $x=(r, \theta) \notin \operatorname{Cut}(p) \cup\{p\}$. Moreover, if equality is achieved at a point, then $m=1$ and at that point $\nabla_{\nabla r_{p}}$ has at most one non-zero eigenvalue which is of multiplicity $n-1$. 
Proof. We modify the proof of the Laplacian comparison theorem on weighted complete Riemannian manifolds with the $\mathrm{CD}(K, 1)$-condition by Wylie and Yeroshkin [35]. The usual Bochner-Weitzenböck formula for functions says that for any $u \in C^{3}(M)$,

$$
\frac{1}{2} \Delta|\nabla u|^{2}=\left|\nabla^{2} u\right|^{2}+\operatorname{Ric}(\nabla u, \nabla u)+\langle\nabla \Delta u, \nabla u\rangle
$$

The Bochner-Weitzenböck formula for the $V$-Laplacian and the $m$-Bakry-Émery Ricci curvature is given by

$$
\begin{aligned}
\frac{1}{2} \Delta_{V}|\nabla u|^{2} & =\left|\nabla^{2} u\right|^{2}+\operatorname{Ric}_{\infty, n}\left(\Delta_{V}\right)(\nabla u, \nabla u)+\left\langle\nabla \Delta_{V} u, \nabla u\right\rangle \\
& =\left|\nabla^{2} u\right|^{2}+\operatorname{Ric}_{m, n}\left(\Delta_{V}\right)(\nabla u, \nabla u)-\frac{V^{*} \otimes V^{*}}{n-m}(\nabla u, \nabla u)+\left\langle\nabla \Delta_{V} u, \nabla u\right\rangle .
\end{aligned}
$$

Consider this equation with $u=r_{p}$ at an interior point of a minimizing geodesic (so that $r_{p}$ is smooth in a neighborhood). Then $\left|\nabla r_{p}\right|=1$ in this neighborhood, so that the left hand side is zero. Now we claim $\nabla_{\nabla r_{p}} \nabla r_{p}=0$ i.e., $\nabla r_{p}$ is a null vector for $\nabla_{\nabla r_{p}}$. For this, it suffices to show that for any smooth vector field $X$ on $M \backslash\{p\}$

$$
\left\langle\nabla_{\nabla r_{p}} \nabla r_{p}, X\right\rangle=0
$$

This is true if $X$ is parallel to $\nabla r_{p}$, because for $f \in C^{\infty}(M \backslash\{p\})$

$$
\left\langle\nabla_{\nabla_{r_{p}}} \nabla r_{p}, f \nabla r_{p}\right\rangle=f\left\langle\nabla_{\nabla_{r_{p}}} \nabla r_{p}, \nabla r_{p}\right\rangle=f \frac{1}{2}\left(\nabla r_{p}\right)\left|\nabla r_{p}\right|^{2}=0
$$

Moreover, (3.3) holds if $X$ is vertical to $\nabla r_{p}$, because

$$
\left\langle\nabla r_{p} \nabla r_{p}, X\right\rangle=\frac{1}{2}\left(\nabla r_{p}\right)\left\langle\nabla r_{p}, X\right\rangle=\frac{1}{2}\left(\nabla r_{p}\right) 0=0
$$

Hence $\nabla_{\nabla r_{p}}$ has at most $n-1$ non-zero eigenvalues and by the Cauchy-Schwarz inequality, it holds on $(\operatorname{Cut}(p) \cup\{p\})^{c}$ that (see [35])

$$
\left|\operatorname{Hess} r_{p}\right|^{2}=\left\|\nabla_{\nabla r_{p}}\right\|^{2} \geq \frac{\left(\Delta r_{p}\right)^{2}}{n-1} .
$$

Now $m \leq 1$. Hence

$$
0 \geq \frac{\left(\Delta r_{p}\right)^{2}}{n-m}+\operatorname{Ric}_{m, n}\left(\Delta_{V}\right)\left(\nabla r_{p}, \nabla r_{p}\right)-\frac{1}{n-m}\left|\left\langle V, \nabla r_{p}\right\rangle\right|^{2}+\left\langle\nabla \Delta_{V} r_{p}, \nabla r_{p}\right\rangle
$$

This gives us the following inequality along $\gamma$,

$$
\frac{\mathrm{d}}{\mathrm{d} r}\left(\Delta_{V} r_{p}\right)(r, \theta) \leq-\frac{\left(\Delta r_{p}(r, \theta)\right)^{2}}{n-m}-\operatorname{Ric}_{m, n}\left(\Delta_{V}\right)\left(\dot{\gamma}_{r}, \dot{\gamma}_{r}\right)+\frac{1}{n-m}\left|\left\langle V, \nabla r_{p}\right\rangle(r, \theta)\right|^{2}
$$


From this, we have

$$
\begin{aligned}
& \frac{\mathrm{d} \lambda}{\mathrm{d} s}=C_{p}^{-1} e^{\frac{2 V_{\gamma}(r)}{n-m}} \frac{\mathrm{d} \lambda}{\mathrm{d} r} \\
&=C_{p}^{-2} e^{\frac{2 V_{\gamma}(r)}{n-m}}\left\{\left(\frac{\mathrm{d}}{\mathrm{d} r} e^{\frac{2 V_{\gamma}(r)}{n-m}}\right) \Delta_{V} r_{p}(r, \theta)+e^{\frac{2 V_{\gamma}(r)}{n-m}} \frac{\mathrm{d}}{\mathrm{d} r} \Delta_{V} r_{p}(r, \theta)\right\} \\
&=C_{p}^{-2} e^{\frac{2 V_{\gamma}(r)}{n-m}}\left\{e^{\frac{2 V_{\gamma}(r)}{n-m}} \frac{2}{n-m} \cdot \frac{\partial V_{\gamma}(r)}{\partial r} \cdot \Delta_{V} r_{p}(r, \theta)+e^{\frac{2 V_{\gamma}(r)}{n-m}} \frac{\mathrm{d}}{\mathrm{d} r} \Delta_{V} r_{p}(r, \theta)\right\} \\
&=C_{p}^{-2} e^{\frac{4 V_{\gamma}(r)}{n-m}}\left\{\frac{2}{n-m} \cdot \frac{\partial V_{\gamma}(r)}{\partial r} \cdot \Delta_{V} r_{p}(r, \theta)+\frac{\mathrm{d}}{\mathrm{d} r} \Delta_{V} r_{p}(r, \theta)\right\} \\
& \leq \frac{C_{p}^{-2}}{n-m} e^{\frac{4 V_{\gamma}(r)}{n-m}}\left\{2 \frac{\partial V_{\gamma}(r)}{\partial r} \Delta_{V} r_{p}(r, \theta)-\left(\Delta r_{p}(r, \theta)\right)^{2}+\left|\left\langle V, \nabla r_{p}\right\rangle(r, \theta)\right|^{2}\right\} \\
&=-\frac{C_{p}^{-2}}{n-m} e^{\frac{4 V_{\gamma}(r)}{n-m}}\left(\Delta_{V} e_{p}(r, \theta)\right)^{2}-C_{p}^{-2} e^{\frac{4 V_{\gamma}(r)}{n-m}} \operatorname{Ric}_{m, n}\left(\Delta_{V}\right)\left(\dot{\gamma}_{r}, \dot{\gamma}_{r}\right) \\
&=-\frac{1}{n-m}\left(C_{p}^{-1} e^{\frac{2 V_{\gamma}(r)}{n-m}} \Delta_{V} r_{p}(r, \theta)\right)^{2}-C_{p}^{-2} e^{\frac{4 V_{\gamma}(r)}{n-m}} \operatorname{Ric}_{m, n}\left(\Delta_{V}\right)\left(\dot{\gamma}_{r}, \dot{\gamma}_{r}\right) \\
&\left.=-\frac{\lambda^{2}}{n-m}-\dot{\gamma}_{r}\right) \\
& C_{p}^{-2} e^{\frac{4 V_{\gamma}(r)}{n-m}} \operatorname{Ric}_{m, n}\left(\Delta_{V}\right)\left(\dot{\gamma}_{r}, \dot{\gamma}_{r}\right) .
\end{aligned}
$$

Here we use (3.6) at the inequality above and use $\Delta_{V} r_{p}=\Delta r_{p}-\left\langle V, \nabla r_{p}\right\rangle$ in the next equality. If the equality holds for (3.1) at some $x=\left(r_{0}, \theta\right) \notin \operatorname{Cut}(p) \cup\{p\}$, then the equality for (3.6) equivalently the equality for (3.5) at $x \notin \operatorname{Cut}(p) \cup\{p\}$ holds, i.e.,

$$
\begin{aligned}
0 & =\frac{\left(\Delta r_{p}\right)^{2}}{n-m}+\operatorname{Ric}_{m, n}\left(\Delta_{V}\right)\left(\nabla r_{p}, \nabla r_{p}\right)-\frac{1}{n-m}\left|\left\langle V, \nabla r_{p}\right\rangle\right|^{2}+\left\langle\nabla \Delta_{V} r_{p}, \nabla r_{p}\right\rangle \\
& \geq \frac{\left(\Delta r_{p}\right)^{2}}{n-1}+\operatorname{Ric}_{m, n}\left(\Delta_{V}\right)\left(\nabla r_{p}, \nabla r_{p}\right)-\frac{1}{n-m}\left|\left\langle V, \nabla r_{p}\right\rangle\right|^{2}+\left\langle\nabla \Delta_{V} r_{p}, \nabla r_{p}\right\rangle
\end{aligned}
$$

holds at $x \notin \operatorname{Cut}(p) \cup\{p\}$. This and $m \leq 1$ yield

$$
\frac{m-1}{(n-m)(n-1)}\left(\Delta r_{p}\right)^{2}(x)=0 \text {. }
$$

Thus $m=1$ or $\Delta r_{p}(x)=0$. Since $M$ has an upper bound $\kappa_{\varepsilon}>0$ of the sectional curvature on some $B_{\varepsilon}(p) \subset \operatorname{Cut}(p)^{c}$, the usual Laplacian comparison theorem tells us that $\Delta r_{p}(x) \geq(n-1) \sqrt{\kappa_{\varepsilon}} \cot \left(\sqrt{\kappa_{\varepsilon}} r_{p}(x)\right)>0$ for $0<r_{p}(x)<\varepsilon$. Therefore we obtain $m=1$, in particular, the equality for (3.4) holds at $x$. This implies that $\nabla_{\nabla r_{p}}$ at $x$ has at most one non-zero eigenvalue of multiplicity $n-1$.

Let $\kappa$ be a continuous function on $[0,+\infty$ [ with respect to the parameter $s$. Assuming the curvature bound $\operatorname{Ric}_{m, n}\left(\Delta_{V}\right)_{x}\left(\nabla r_{p}, \nabla r_{p}\right) \geq(n-m) \kappa\left(s_{p}(x)\right) e^{-\frac{4 \phi_{V}(x)}{n-m}} C_{p}^{2}$ for $s_{p}(x)<S$ with $x \notin \operatorname{Cut}(p) \cup\{p\}$, we see $\operatorname{Ric}_{m, n}\left(\Delta_{V}\right)\left(\dot{\gamma}_{r}, \dot{\gamma}_{r}\right) \geq(n-m) \kappa(s) e^{-\frac{4 V_{\gamma}(r)}{n-m}} C_{p}^{2}$ for $s=$ 
$s(r, \theta)<S$ with $0<r<d(p, \operatorname{Cut}(p))$. From (3.1) we have the usual Riccati inequality

$$
\left.-\frac{\mathrm{d} \lambda}{\mathrm{d} s}(s) \geq(n-m) \kappa(s)+\frac{\lambda(s)^{2}}{n-m} \quad \text { for } \quad s \in\right] 0, S[
$$

with the caveat that it is in terms of the parameter $s$ instead of $r$. This gives us the following comparison estimate.

LEMma 3.2. Suppose that $(M, g)$ be an $n$-dimensional complete Riemannian manifold and $V$ a $C^{1}$-vector field. Fix $\left.R \in\right] 0,+\infty[$ and $x, p \in M$. Assume that (2.9) holds for $r_{p}(x)<R$ with $x \notin(\operatorname{Cut}(p) \cup\{p\})$. Let $\gamma, s$, and $\lambda$ be defined to be as in Lemma [3.1. Then

$$
\lambda(r, \theta) \leq m_{\kappa}(s)
$$

holds for $r<R, s<\delta_{\kappa}$ and $x=(r, \theta) \notin \operatorname{Cut}(p) \cup\{p\}$. Here

$$
s=s_{p}(r)=C_{p} \int_{0}^{r} \exp \left(-\frac{2 \phi_{V}\left(\gamma_{t}\right)}{n-m}\right) \mathrm{d} t .
$$

Suppose further that the equality in (3.8) holds for some $r_{0}<R$ with $s_{0}:=s\left(r_{0}\right)<\delta_{\kappa}$. We choose an orthonormal basis $\left\{e_{i}\right\}_{i=1}^{n}$ of $T_{p} M$ with $e_{n}=\dot{\gamma}_{0}$. Let $\left\{Y_{i}\right\}_{i=1}^{n-1}$ be the Jacobi fields along $\gamma$ with $Y_{i}(0)=o_{p}$ and $Y_{i}^{\prime}(0)=e_{i}$. Then we have $m=1$, and at $x=(r, \theta)$ with $r \leq r_{0}, \nabla_{\nabla r_{p}}$ has at most one non-zero eigenvalue which is of multiplicity $n-1$, and for all $\left.r \in] 0, r_{0}\right]$ we have

$$
\operatorname{Ric}_{1, n}\left(\Delta_{V}\right)\left(\dot{\gamma}_{r}, \dot{\gamma}_{r}\right)=(n-1) \kappa\left(s_{p}\left(\gamma_{r}\right)\right) e^{-\frac{4 V_{\gamma}(r)}{n-1}} C_{p}^{2}
$$

Moreover, for all $i$ we have $Y_{i}(r)=C_{p}^{-1} F_{\kappa}(r) E_{i}(r)$ for $r \in\left[0, r_{0}\right]$, where

$$
F_{\kappa}(r):=\exp \left(\frac{V_{\gamma}(r)}{n-1}\right) \mathfrak{s}_{\kappa}\left(s_{p}\left(\gamma_{r}\right)\right),
$$

and $\left\{E_{i}(r)\right\}_{i=1}^{n-1}$ are the parallel vector fields with $E_{i}(0)=e_{i}$. Consequently,

$$
g_{\gamma_{r}}=d r^{2}+C_{p}^{-2} e^{\frac{2 V_{\gamma}(r)}{n-1}} \mathfrak{s}_{\kappa}^{2}\left(s_{p}\left(\gamma_{r}\right)\right) g_{\mathbb{S}^{n-1}}
$$

Here $g_{\mathbb{S}^{n-1}}$ is the standard metric on the sphere $\mathbb{S}^{n-1}$.

Proof. Set $S:=s_{p}(R)$. Then $r<R$ implies $s<S$. Since $\Delta r_{p}(r, \theta) \rightarrow+\infty$ as $r \rightarrow 0$, we see $\lambda(r, \theta) \rightarrow+\infty$ as $r \rightarrow 0$ or $s \rightarrow 0$. We set $\beta(s):=\mathfrak{s}_{\kappa}^{2}(s)\left(\lambda-m_{\kappa}(s)\right)$. Then, by 
(2.8) and (3.7), for $s<S$

$$
\begin{aligned}
\beta^{\prime}(s) & =2 \mathfrak{s}_{\kappa}^{\prime}(s) \mathfrak{s}_{\kappa}(s)\left(\lambda-m_{\kappa}(s)\right)+\mathfrak{s}_{\kappa}^{2}(s)\left(\frac{\mathrm{d} \lambda}{\mathrm{d} s}-m_{\kappa}^{\prime}(s)\right) \\
& =2 \mathfrak{s}_{\kappa}^{2}(s) \cot _{\kappa}(s)\left(\lambda-m_{\kappa}(s)\right)+\mathfrak{s}_{\kappa}^{2}(s)\left(\frac{\mathrm{d} \lambda}{\mathrm{d} s}+(n-m) \kappa(s)+\frac{m_{\kappa}^{2}(s)}{n-m}\right) \\
& \leq \frac{\mathfrak{s}_{\kappa}^{2}(s)}{n-m}\left(2 m_{\kappa}(s) \lambda-2 m_{\kappa}^{2}(s)\right)+\frac{\mathfrak{s}_{\kappa}^{2}(s)}{n-m}\left(m_{\kappa}^{2}(s)-\lambda^{2}\right) \\
& =-\frac{\mathfrak{s}_{\kappa}^{2}(s)}{n-m}\left(\lambda-m_{\kappa}(s)\right)^{2} \leq 0 .
\end{aligned}
$$

We note here that (3.7) is derived from (2.9). If we show $\beta(0)=0$, then $\beta(s) \leq \beta(0)=0$. For this, it suffices to prove that $s\left(\lambda-m_{\kappa}(s)\right)$ is upper bounded as $s \rightarrow 0$. We already know that $\lim _{s \rightarrow 0} s m_{\kappa}(s)=n-m$ and the ratio $s / r=s_{p}(r) / r$ converges to $C_{p}$ as $r \rightarrow 0$. So it suffices to prove $\lim _{r \rightarrow 0} r \lambda(r, \theta)=C_{p}^{-1}(n-1)$ as $r \rightarrow 0$, equivalently $\lim _{r \rightarrow 0} r \Delta r_{p}(r, \theta)=n-1$, because $\lim _{r \rightarrow 0} r\left\langle V, \nabla r_{p}\right\rangle(r, \theta)=0$. In view of the usual Laplacian comparison theorem for the Laplace-Bertrami operator $\Delta$ under the upper (resp. lower) bound $K_{\varepsilon}$ (resp. $\kappa_{\varepsilon}$ ) of sectional curvature on $B_{\varepsilon}(p)$, we see $(n-1) \cot _{K_{\varepsilon}}(r) \leq$ $\Delta r_{p}(r, \theta) \leq(n-1) \cot _{\kappa_{\varepsilon}}(r)$ on $B_{\varepsilon}(p)$. This implies the desired assertion. Next we assume that the equality in (3.8) holds for some $r_{0}<R$, i.e., $\lambda\left(r_{0}, \theta\right)=(n-m) \cot _{\kappa}\left(s_{0}\right)$ for $r_{0}<R$ with $s_{0}=s\left(r_{0}\right)$. This implies $0=\beta\left(s_{0}\right) \leq \beta(s) \leq \beta(0)=0$, hence $\lambda(r)=m_{\kappa}(s)$ for all $s \in\left[0, s_{0}\right]$. From this,

$$
\frac{\mathrm{d} \lambda}{\mathrm{d} s}\left(s_{0}\right)=\frac{\mathrm{d} m_{\kappa}}{\mathrm{d} s}\left(s_{0}\right)
$$

In particular, we have at $r_{0}$

$$
\begin{aligned}
\frac{\mathrm{d} \lambda}{\mathrm{d} s} & \leq-\frac{\lambda(r)^{2}}{n-m}-C_{p}^{-2} e^{\frac{4 V_{\gamma}(r)}{n-m}} \operatorname{Ric}_{m, n}\left(\Delta_{V}\right)\left(\dot{\gamma}_{r}, \dot{\gamma}_{r}\right) \\
& \leq-\frac{\lambda(r)^{2}}{n-m}-(n-m) \kappa(s)=-\frac{m_{\kappa}(s)^{2}}{n-m}-(n-m) \kappa(s)=\frac{\mathrm{d} \lambda}{\mathrm{d} s} .
\end{aligned}
$$

Then the equality holds in (3.1) at $x=\left(r_{0}, \theta\right)$. So we have $m=1$ by Lemma [3.1, We can conclude $\beta(s) \equiv 0$ on $\left[0, s_{0}\right]$ from $\beta(0)=\beta\left(s_{0}\right)=0$ and $\beta^{\prime}(s) \leq 0$ so that $\lambda(r, \theta)=(n-1) \cot _{\kappa}(s)$ for $\left.\left.s \in\right] 0, s_{0}\right]$. We then see the equality (3.12) at any $\left.\left.r \in\right] 0, r_{0}\right]$, hence (3.9) holds at any $\left.r \in] 0, r_{0}\right]$.

Finally we prove (3.11) at any $\left.r \in] 0, r_{0}\right]$ under $\lambda\left(r_{0}\right)=(n-m) \cot _{\kappa}\left(s_{0}\right)$. Hereafter, we assume $\left.r \in] 0, r_{0}\right]$. By Lemma 3.1, at $x=(r, \theta), \nabla_{\nabla r_{p}}$ has a non-zero eigenvalue $A(r)$ which is of $n-1$ multiplicity. Then we have

$$
\begin{aligned}
\lambda(r, \theta) & =C_{p}^{-1} e^{\frac{2 V_{\gamma}(r)}{n-1}}\left(\Delta r_{p}(r, \theta)-\left\langle V, \nabla r_{p}\right\rangle(r, \theta)\right) \\
& =C_{p}^{-1} e^{\frac{2 V_{\gamma}(r)}{n-1}}\left((n-1) A(r)-\left\langle V, \nabla r_{p}\right\rangle(r, \theta)\right)=(n-1) \cot _{\kappa}(s),
\end{aligned}
$$


where we use the equality (3.8) at any $\left.r \in] 0, r_{0}\right]$. So we have $A(r)=C_{p} e^{-\frac{2 V_{\gamma}(r)}{n-1}} \cot _{\kappa}(s)+$ $\frac{\left\langle V, \nabla r_{p}\right\rangle(r, \theta)}{n-1}=(n-1)^{-1} \Delta r_{p}\left(\gamma_{r}\right)$. The radial curvature equation (see [26, Theorem 2 in pp. 44]) tells us that

$$
R\left(E_{i}, \dot{\gamma}_{r}\right) \dot{\gamma}_{r}=-\left(A^{\prime}(r)+A(r)^{2}\right) E_{i}
$$

Combining Bochner-Weitzenböck formula with (3.9), we have

$$
A^{\prime}(r)+A(r)^{2}=\frac{V_{\gamma}^{\prime \prime}(r)}{n-1}+\left(\frac{V_{\gamma}^{\prime}(r)}{n-1}\right)^{2}-\kappa\left(s_{p}\left(\gamma_{r}\right)\right) e^{-\frac{4 V_{\gamma}(r)}{n-1}} C_{p}^{2}=\frac{F_{\kappa}^{\prime \prime}(r)}{F_{\kappa}(r)}
$$

Since $F_{\kappa}(0)=0$ and $F_{\kappa}^{\prime}(0)=C_{p}$, we obtain

$$
Y_{i}(r)=C_{p}^{-1} F_{\kappa}(r) E_{i}(r)=C_{p}^{-1} e^{\frac{V_{\gamma}(r)}{n-1}} \mathfrak{s}_{\kappa}\left(s_{p}\left(\gamma_{r}\right)\right) E_{i}(r) .
$$

This proves the desired conclusion.

Corollary 3.3. Let $(M, g)$ be an $n$-dimensional complete Riemannian manifold and $V$ a $C^{1}$-vector field. Fix $p \in M$ and $\left.R \in\right] 0,+\infty\left[\right.$. Assume that (2.9) holds for $r_{p}(x)<R$ with $x \notin \operatorname{Cut}(p) \cup\{p\}$. Then $s_{p}(x)<\delta_{\kappa}$.

Proof. We may assume $\delta_{\kappa}<\infty$. Take $x \in B_{R}(p)$ with $x \notin \operatorname{Cut}(p) \cup\{p\}$. Let $x=(r, \theta)$ be the polar coordinate expression around $p$ and set $s:=s_{p}(r)=C_{p} \int_{0}^{r} \exp \left(-\frac{2 \phi_{V}\left(\gamma_{t}\right)}{n-m}\right) \mathrm{d} t$ and $S=s_{p}(R)$, where $\gamma$ is a unit speed geodesic with $\gamma_{0}=p$ and $\dot{\gamma}_{0}=\theta$. We see $s_{p}(x)<S$. Assume $S>\delta_{\kappa}$. Then there exists $\left.r_{0} \in\right] 0, R\left[\right.$ such that $\delta_{\kappa}=C_{p} \int_{0}^{r_{0}} \exp \left(-\frac{2 V_{\gamma}(t)}{n-m}\right) \mathrm{d} t$. By (3.8), $\lambda(r, \theta) \leq(n-m) \cot _{\kappa}(s)$ holds for $s<\delta_{\kappa}$. Since $r \uparrow r_{0}$ is equivalent to $s=s(r) \uparrow \delta_{\kappa}$, we have

$$
\lambda\left(r_{0}, \theta\right)=\lim _{r \uparrow r_{0}} \lambda(r, \theta) \leq \lim _{r \uparrow r_{0}}(n-m) \cot _{\kappa}(s(r))=-\infty .
$$

This contradicts the well-definedness of $\lambda(r, \theta)=C_{p}^{-1}\left(e^{\frac{2 \phi_{V}}{n-m}} \Delta_{V} r_{p}\right)(r, \theta)$ for $\left.r \in\right] 0, R[$. Therefore $S \leq \delta_{\kappa}$ under $\delta_{\kappa}<\infty$ and we obtain the conclusion $s_{p}(x)<S \leq \delta_{\kappa}$.

Let $p \in M$ and let $(r, \theta), r>0, \theta \in \mathbb{S}^{n-1}$ be exponential polar coordinates (for the metric $g$ ) around $p$ which are defined on a maximal star shaped domain in $T_{p} M$ called the segment domain. Write the volume element $\mathrm{d} \mathfrak{m}=J(r, \theta) \mathrm{d} r \wedge \mathrm{d} \theta$.

Let $s_{p}(\cdot)$ be the re-parametrized distance function defined above. Inside the segment domain, $s_{p}$ has the simple formula

$$
s_{p}(r, \theta)=C_{p} \int_{0}^{r} e^{-\frac{2 \phi_{V}(t, \theta)}{n-m}} \mathrm{~d} t .
$$

Therefore, $s_{p}$ is a smooth function in the segment domain with the property that $\frac{\partial s}{\partial r}=$ $C_{p} e^{-\frac{2 \phi_{V}(r, \theta)}{n-m}}$. We can then also take $(s, \theta)$ to be coordinates which are also valid for the entire segment theorem. We can not control the derivative of $s$ in directions tangent to 
the sphere, so the new $(s, \theta)$ coordinates are not orthogonal as in the case for geodesic polar coordinates. However, this is not the issue when we computing volumes as

$$
\begin{aligned}
e^{-\frac{2 \phi_{V}}{n-m}} \mathrm{~d} \mu_{V} & =e^{-\frac{n-m+2}{n-m} \phi_{V}} J(r, \theta) \mathrm{d} r \wedge \mathrm{d} \theta \\
& =C_{p}^{-1} e^{-\phi_{V}} J(r, \theta) \mathrm{d} s \wedge \mathrm{d} \theta
\end{aligned}
$$

Here $\mathrm{d} \mu_{V}=e^{-\phi_{V}} \mathrm{dm}$. We denote the derivative in the radial direction in terms of this

parameter by $\frac{\mathrm{d}}{\mathrm{d} s}$. In geodesic polar coordinates $\frac{\mathrm{d}}{\mathrm{d} s}$ has the expression $\frac{\mathrm{d}}{\mathrm{d} s}=C_{p}^{-1} e^{\frac{2 \phi_{V}(r, \theta)}{n-m}} \frac{\partial}{\partial r}$. Note that it is not the same as $\frac{\partial}{\partial s}$ in $(s, \theta)$ coordinates.

Proof of Theorem 2.5. The implication (2.9) $\Longrightarrow(2.10)$ for $R<\infty$ follows from Lemma 3.2 , because $r_{p}$ is smooth on $M \backslash(\operatorname{Cut}(p) \cup\{p\})$. The implication (2.9) $\Longrightarrow(2.10)$ for $R=+\infty$ follows from it.

\section{Proofs of Theorem 2.10] and Corollary 2.11}

Proof Theorem 2.10. Suppose that there exist points $p, q \in M$ such that $s(p, q)>\delta_{\kappa}$. Since $\operatorname{Cut}(p)$ is closed and measure zero, we may assume $q \notin \operatorname{Cut}(p)$. By Lemma 3.2, along minimal geodesic from $p$ to $q, \lambda(r, \theta) \leq m_{\kappa}(s)$. However, as $s \rightarrow \delta_{\kappa}, m_{\kappa}(s) \rightarrow-\infty$. This implies $\Delta r_{p}(x) \rightarrow-\infty$ as $s(p, x) \rightarrow \delta_{\kappa}$. This contradicts that $r_{p}$ is smooth in a neighborhood of $q$. The final assertion follows Remark 2.4.

Proof of Corollary 2.11. Suppose that $\sup _{q \in M} d(p, q)=+\infty$. Then there exists a sequence $\left\{q_{i}\right\}$ in $M$ such that $d\left(p, q_{i}\right) \rightarrow+\infty$ as $i \rightarrow+\infty$. By Lemma 2.3, $s\left(p, q_{i}\right) \rightarrow+\infty$ as $k \rightarrow+\infty$, which contradicts $\sup _{q \in M} s(p, q) \leq \delta_{\kappa}$. Therefore, $\sup _{q \in M} d(p, q)<\infty$, hence $M$ is compact.

\section{Proof of Theorem 2.13}

Recall that for a Riemannian manifold $\frac{\mathrm{d}}{\mathrm{d} r} \log J(r, \theta)=\Delta r_{p}(r, \theta)$, where $\Delta r_{p}$ is the standard Laplacian acting on the distance function $r_{p}$ from the point $p$. (3.15) indicates we should consider the quantity

$$
\frac{\mathrm{d}}{\mathrm{d} s} \log \left(e^{-V_{\gamma}(r)} J(r, \theta)\right)=C_{p}^{-1} e^{\frac{2 V_{\gamma}(r)}{n-m}}\left(\Delta r_{p}(r, \theta)-\left\langle V_{\gamma_{r}}, \dot{\gamma}_{r}\right\rangle\right)=C_{p}^{-1} e^{\frac{2 V_{\gamma}(r)}{n-m}} \Delta_{V} r_{p}(r, \theta)
$$

Lemma 5.1 (Volume Element Comparison). Let $(M, g)$ be an $n$-dimensional complete Riemannian manifold and $V$ a $C^{1}$-vector field. Fix $p \in M$ and $\left.\left.R \in\right] 0,+\infty\right]$. Assume that (2.9) holds for $r_{p}(x)<R$ with $x \notin \operatorname{Cut}(p) \cup\{p\}$. Let $J$ be the volume element in geodesic polar coordinates around $p \in M$ and set $J_{V}(r, \theta):=e^{-V_{\gamma}(r)} J(r, \theta)$. Then for $r_{0}<r_{1}<R$ with $r_{1}<\operatorname{cut}(\theta)$,

$$
\frac{J_{V}\left(r_{1}, \theta\right)}{J_{V}\left(r_{0}, \theta\right)} \leq \frac{\mathfrak{s}_{\kappa}\left(s_{p}\left(r_{1}, \theta\right)\right)^{n-m}}{\mathfrak{s}_{\kappa}\left(s_{p}\left(r_{0}, \theta\right)\right)^{n-m}} .
$$

Here $\operatorname{cut}(\theta)$ is the distance from $p$ to the cut point along the geodesic with $\gamma(0)=p$ and $\dot{\gamma}(0)=\theta$. 
Proof. Recall $s=s_{p}(r)=s_{p}(r, \theta)=C_{p} \int_{0}^{r} \exp \left(-\frac{2 V_{\gamma}(t)}{n-m}\right) \mathrm{d} t$ and $\gamma$ is the unit speed geodesic from $p$ with $\dot{\gamma}_{0}=\theta$. First note that the right hand side of (5.2) is meaningful for $r_{0}<r_{1}<R$. Indeed, if $R<+\infty, s_{p}\left(r_{0}, \theta\right)<s_{p}\left(r_{1}, \theta\right)<\delta_{\kappa}$ by Corollary 3.3. If $R=+\infty$, we can take $\left.R_{0} \in\right] r_{1},+\infty$ [ so that (2.9) holds for $r_{p}(x)<R_{0}$, hence $s_{p}\left(r_{0}, \theta\right)<s_{p}\left(r_{1}, \theta\right)<$ $\delta_{\kappa}$ by Corollary 3.3. From Lemma 3.2 and (5.1) we have that

$$
\frac{\mathrm{d}}{\mathrm{d} s} \log J_{V}(r, \theta)=C_{p}^{-1} e^{\frac{2 \phi_{V}}{n-m}} \Delta_{V} r_{p}(r, \theta) \leq(n-m) \cot _{\kappa}(s)=\frac{\mathrm{d}}{\mathrm{d} s} \log \left(\mathfrak{s}_{\kappa}(s)^{n-m}\right)
$$

for $r \in] 0, R \wedge \operatorname{cut}(\theta)\left[\right.$. Integrating (5.3) between any $s_{0}<s_{1}$ with $s_{i}=s_{p}\left(r_{i}, \theta\right)$ and $\left.r_{i} \in\right] 0, R \wedge \operatorname{cut}(\theta)[(i=0,1)$ gives

$$
\log \left(\frac{J_{V}\left(r_{1}, \theta\right)}{J_{V}\left(r_{0}, \theta\right)}\right) \leq \log \left(\frac{\mathfrak{s}_{\kappa}\left(s_{1}\right)^{n-m}}{\mathfrak{s}_{\kappa}\left(s_{0}\right)^{n-m}}\right) \quad \text { implies } \quad \frac{J_{V}\left(r_{1}, \theta\right)}{J_{V}\left(r_{0}, \theta\right)} \leq \frac{\mathfrak{s}_{\kappa}\left(s_{1}\right)^{n-m}}{\mathfrak{s}_{\kappa}\left(s_{0}\right)^{n-m}}
$$

for all $r_{0}<r_{1}<R \wedge \operatorname{cut}(\theta)$. Note that since $\mathrm{d} s$ is an orientation preserving change of variables along the geodesic $\gamma$, the quantity is also non-increasing in terms of the parameter $r \in] 0, R \wedge \operatorname{cut}(\theta)[$.

Proof of Theorem 2.13. By Lemma 5.1, for all $r_{1}, r_{2}>0$ with $r_{1}<r_{2}<R$ and $r_{2}<\operatorname{cut}(\theta)$

$$
\frac{J_{V}\left(r_{2}, \theta\right)}{J_{V}\left(r_{1}, \theta\right)} \leq \frac{\mathfrak{s}_{\kappa}^{n-m}\left(s_{p}\left(r_{2}, \theta\right)\right)}{\mathfrak{s}_{\kappa}^{n-m}\left(s_{p}\left(r_{1}, \theta\right)\right)} \leq \frac{\mathfrak{s}_{\kappa}^{n-m}\left(\sup _{\eta \in \mathbb{S}^{n-1}} s_{p}\left(r_{2}, \eta\right)\right)}{\mathfrak{s}_{\kappa}^{n-m}\left(\inf _{\eta \in \mathbb{S}^{n-1}} s_{p}\left(r_{1}, \eta\right)\right)}
$$

So for $0 \leq r_{a}<r_{b} \leq r_{d}, 0 \leq r_{a} \leq r_{c}<r_{d}$ and $r_{d}<R$, we have following inequality

$$
\begin{aligned}
\frac{\int_{\operatorname{cut}(\theta) \wedge r_{c}}^{\operatorname{cut}(\theta) \wedge r_{d}} J_{V}\left(r_{2}, \theta\right) \mathrm{d} r_{2}}{\int_{\operatorname{cut}(\theta) \wedge r_{a}}^{\operatorname{cut}(\theta) r_{b}} J_{V}\left(r_{1}, \theta\right) \mathrm{d} r_{1}} & \leq \frac{\int_{\operatorname{cut}(\theta) \wedge r_{c}}^{\operatorname{cut}(\theta) \wedge r_{d}} \mathfrak{s}_{\kappa}^{n-m}\left(s_{p}\left(r_{2}, \theta\right)\right) \mathrm{d} r_{2}}{\int_{\operatorname{cut}(\theta) \wedge r_{b}}^{\operatorname{cut}(\theta)} \mathfrak{s}_{\kappa}^{n-m}\left(s_{p}\left(r_{1}, \theta\right)\right) \mathrm{d} r_{1}} \\
& \leq \frac{\int_{r_{c}}^{r_{d}} \mathfrak{s}_{\kappa}^{n-m}\left(\sup _{\eta \in \mathbb{S}^{n-1}} s_{p}\left(r_{2}, \eta\right)\right) \mathrm{d} r_{2}}{\int_{r_{a}}^{r_{b}} \mathfrak{s}_{\kappa}^{n-m}\left(\inf _{\eta \in \mathbb{S}^{n-1}} s_{p}\left(r_{1}, \eta\right)\right) \mathrm{d} r_{1}}
\end{aligned}
$$

under $r_{a}=r_{c}$ or $r_{b}=r_{d}$ by use of [36, Lemma 3.1] (cf. [36, Proof of Theorem 3.2]). From this, we can deduce that

$$
\frac{\int_{\mathbb{S}^{n-1}} \int_{\operatorname{cut}(\theta) \wedge r_{c}}^{\operatorname{cut}(\theta) \wedge r_{d}} J_{V}\left(r_{2}, \theta\right) \mathrm{d} r_{2} \mathrm{~d} \theta}{\int_{\mathbb{S}^{n-1}} \int_{\operatorname{cut}(\theta) \wedge r_{a}}^{\operatorname{cut}(\theta) r_{b}} J_{V}\left(r_{1}, \theta\right) \mathrm{d} r_{1} \mathrm{~d} \theta} \leq \frac{\int_{\mathbb{S}^{n-1}} \int_{r_{c}}^{r_{d}} \mathfrak{s}_{\kappa}^{n-m}\left(\sup _{\eta \in \mathbb{S}^{n-1}} s_{p}\left(r_{2}, \eta\right)\right) \mathrm{d} r_{2} \mathrm{~d} \theta}{\int_{\mathbb{S}^{n-1}} \int_{r_{a}}^{r_{b}} \mathfrak{s}_{\kappa}^{n-m}\left(\inf _{\eta \in \mathbb{S}^{n-1}} s_{p}\left(r_{1}, \eta\right)\right) \mathrm{d} r_{1} \mathrm{~d} \theta}
$$

holds for general $0 \leq r_{a}<r_{b} \leq r_{d}, 0 \leq r_{a} \leq r_{c}<r_{d}$ and $r_{d}<R$. This implies that (2.17) holds for $r_{1}<R$. If $\phi$ is rotationally symmetric around $p, s_{p}(r, \theta)$ can be written as $s_{p}(r)$ and one can derive

$$
\frac{\int_{\mathbb{S}^{n-1}} \int_{\operatorname{cut}(\theta) \wedge r_{c}}^{\operatorname{cut}(\theta) \wedge r_{d}} J_{V}\left(r_{2}, \theta\right) \mathrm{d} r_{2} \mathrm{~d} \theta}{\int_{\mathbb{S}^{n-1}} \int_{\operatorname{cut}(\theta) \wedge r_{a}}^{\operatorname{cut}(\theta) \wedge r_{b}} J_{V}\left(r_{1}, \theta\right) \mathrm{d} r_{1} \mathrm{~d} \theta} \leq \frac{\int_{\mathbb{S}^{n-1}} \int_{r_{c}}^{r_{d}} \mathfrak{s}_{\kappa}^{n-m}\left(s_{p}\left(r_{2}\right)\right) \mathrm{d} r_{2} \mathrm{~d} \theta}{\int_{\mathbb{S}^{n-1}} \int_{r_{a}}^{r_{b}} \mathfrak{s}_{\kappa}^{n-m}\left(s_{p}\left(r_{1}\right)\right) \mathrm{d} r_{1} \mathrm{~d} \theta} .
$$


This implies that (2.18) holds for $r_{1}<R$. Similarly, in the modified coordinates $(s, \theta)$, we set

$$
\operatorname{cut}_{s}(\theta):=\int_{0}^{\operatorname{cut}(\theta)} e^{-\frac{2 V_{\gamma}(t)}{n-m}} \mathrm{~d} t
$$

where $\gamma$ is the unit speed geodesic with $\gamma_{0}=p$ and $\dot{\gamma}_{0}=\theta$. Then we have

$$
\nu_{V}\left(C\left(p, s_{0}, s_{1}\right)\right)=\int_{\mathbb{S}^{n-1}} \int_{\operatorname{cut}_{s}(\theta) \wedge s_{0}}^{\operatorname{cut}_{s}(\theta) \wedge s_{1}} J_{V}(r(s, \theta), \theta) \mathrm{d} s \mathrm{~d} \theta,
$$

and

$$
v\left(\kappa, s_{0}, s_{1}\right)=\int_{\mathbb{S}^{n-1}} \int_{s_{0}}^{s_{1}} \mathfrak{s}_{\kappa}^{n-m}(s) \mathrm{d} s \mathrm{~d} \theta=\omega_{n-1} \int_{s_{0}}^{s_{1}} \mathfrak{s}_{\kappa}^{n-m}(s) \mathrm{d} s .
$$

Therefore, (21) follows. Here $r(s, \theta):=C_{p}^{-1} \int_{0}^{s} \exp \left(\frac{2 V_{\gamma}\left(f^{-1}(u)\right)}{n-m}\right) \mathrm{d} u$ with $f(r):=s_{p}(r, \theta)$. Note that $s_{1}<\delta_{\kappa}$ always holds under the condition. Indeed, $s_{1}<S$ implies $s_{1}<\delta_{\kappa}$ under $R<+\infty$ by Corollary 3.3. When $R=+\infty$, for any $\theta \in \mathbb{S}^{n-1}$ there exists $\left.R_{0} \in\right] 0,+\infty[$ depending on $\theta$ such that $s_{1}<s\left(R_{0}, \theta\right)$. Then applying Corollary 3.3 for $R_{0}<\infty$, $r_{1}:=r\left(s_{1}, \theta\right)<r\left(s\left(R_{0}, \theta\right), \theta\right)=R_{0}$ implies $s_{1}=s\left(r\left(s_{1}, \theta\right), \theta\right)<\delta_{\kappa}$, where we use (2.9) holds for $r_{p}(x)<R_{0}$.

Proof of Corollary 2.15. By Theorem 2.13)(11), for $0<r_{1}<r_{2}<R$

$$
\begin{aligned}
\frac{\mu_{V}\left(B_{r_{2}}(p)\right)}{\mu_{V}\left(B_{r_{1}}(p)\right)} & \leq \frac{\int_{0}^{r_{2}}\left(C_{p} e^{-\frac{2 \phi_{V}(r)}{n-m}} r\right)^{n-m} \mathrm{~d} r}{\int_{0}^{r_{1}}\left(C_{p} e^{-\frac{2 \bar{\Phi}_{V}(r)}{n-m}} r\right)^{n-m} \mathrm{~d} r} \\
& \leq e^{2\left(\bar{\phi}_{V}\left(r_{1}\right)-\underline{\phi}_{V}\left(r_{2}\right)\right)} \frac{\int_{0}^{r_{2}} r^{n-m} \mathrm{~d} r}{\int_{0}^{r_{1}} r^{n-m} \mathrm{~d} r}=e^{2\left(\bar{\phi}_{V}\left(r_{1}\right)-\underline{\phi}_{V}\left(r_{2}\right)\right)}\left(\frac{r_{2}}{r_{1}}\right)^{n-m+1} .
\end{aligned}
$$

\section{Proofs of Theorem 2.16, Corollaries 2.17 and 2.18}

Proof of Theorem 2.16. Suppose that $M$ is non-compact. Then there exists a unit speed geodesic $\gamma$ with $\gamma_{0}=p$ satisfying (2.23). Note that the function $\lambda(t)$ is smooth for all $t>0$ along $\gamma$. By (3.2), we have

$$
\lambda(t)-\lambda(1)+\frac{C_{p}}{n-m} \int_{1}^{t} e^{-\frac{2 V_{\gamma}(r)}{n-m}} \lambda(r)^{2} \mathrm{~d} r \leq-C_{p}^{-1} \int_{1}^{t} e^{\frac{2 V_{\gamma}(r)}{n-m}} \operatorname{Ric}_{m, n}\left(\Delta_{V}\right)\left(\dot{\gamma}_{r}, \dot{\gamma}_{r}\right) \mathrm{d} r .
$$

Hence

$$
\lim _{t \rightarrow+\infty}\left(\lambda(t)+\frac{C_{p}}{n-m} \int_{1}^{t} e^{-\frac{2 V_{\gamma}(r)}{n-m}} \lambda(r)^{2} \mathrm{~d} r\right)=-\infty .
$$


In particular, $\lim _{t \rightarrow+\infty} \lambda(t)=-\infty$. Next we prove that there exists a finite number $T>0$ such that $\lim _{t \rightarrow T-} \lambda(t)=-\infty$, which contradicts the smoothness of $\lambda(r)$. By (6.1), given $C>n-m$ there exists $t_{0}>1$ such that

$$
-\lambda\left(t_{0}\right)-\frac{C_{p}}{n-m} \int_{1}^{t_{0}} e^{-\frac{2 V_{\gamma}(r)}{n-m}} \lambda(r)^{2} \mathrm{~d} r \geq \frac{C}{n-m}
$$

Since

$$
\lim _{t \rightarrow+\infty} \int_{1}^{t} e^{\frac{2 V_{\gamma}(r)}{n-m}} \operatorname{Ric}_{m, n}\left(\Delta_{V}\right)\left(\dot{\gamma}_{r}, \dot{\gamma}_{r}\right) \mathrm{d} r=+\infty
$$

there exists $\left.t_{1} \in\right] t_{0},+\infty\left[\right.$ such that $\int_{t_{0}}^{t} e^{\frac{2 V_{\gamma}(r)}{n-m}} \operatorname{Ric}_{m, n}\left(\Delta_{V}\right)\left(\dot{\gamma}_{r}, \dot{\gamma}_{r}\right) \mathrm{d} r \geq 0$ for all $t \geq t_{1}$. Let $\psi(t)$ be the function defined by

$$
\psi(t):=-\lambda(t)-\frac{C_{p}}{n-m} \int_{1}^{t} e^{-\frac{2 V_{\gamma}(r)}{n-m}} \lambda(r)^{2} \mathrm{~d} r-C_{p}^{-1} \int_{1}^{t} e^{\frac{2 V_{\gamma}(r)}{n-m}} \operatorname{Ric}_{m, n}\left(\Delta_{V}\right)\left(\dot{\gamma}_{r}, \dot{\gamma}_{r}\right) \mathrm{d} r .
$$

Then we see $\psi^{\prime}(t) \geq 0$ by (3.2). Hence $\psi(t) \geq \psi\left(t_{0}\right)$ for $t \geq t_{1}>t_{0}$. This implies that

$$
-\lambda(t)-\frac{C_{p}}{n-m} \int_{1}^{t} e^{-\frac{2 V_{\gamma}(r)}{n-m}} \lambda(r)^{2} \mathrm{~d} r \geq \frac{C}{n-m}>1
$$

holds for all $t \geq t_{1}$. Let us consider the sequence $\left\{t_{\ell}\right\}$ defined inductively by

$$
C_{p} \int_{t_{\ell}}^{t_{\ell+1}} e^{-\frac{2 V_{\gamma}(r)}{n-m}} \mathrm{~d} r=(n-m)\left(\frac{n-m}{C}\right)^{\ell-1} \quad \text { for } \quad \ell \geq 1 .
$$

The existence of such sequence is guaranteed by the $(V, m)$-completeness of $(M, g, V)$ at $p$. Let $T$ be the increasing limit of $\left\{t_{\ell}\right\}$. Then we see

$$
C_{p} \int_{t_{1}}^{T} e^{-\frac{2 V_{\gamma}(r)}{n-m}} \mathrm{~d} r=\frac{C(n-m)}{C-n+m}
$$

In view of the $(V, m)$-completeness of $(M, g, V)$ at $p$, we have

$$
\int_{1}^{\infty} e^{-\frac{2 V_{\gamma}(r)}{n-m}} \mathrm{~d} r=+\infty .
$$

Thus we obtain $T<\infty$. Finally we claim that for given $\ell \in \mathbb{N},-\lambda(t) \geq\left(\frac{C}{n-m}\right)^{\ell}$ for all $t \geq t_{\ell}$. This is true for $\ell=1$ by (6.3). Suppose that $-\lambda(r) \geq\left(\frac{C}{n-m}\right)^{\ell}$ for all $r \geq t_{\ell}$ and fix $t \geq t_{\ell+1}$. Then using inequality (6.3) again,

$$
\begin{aligned}
-\lambda(t) & \geq \frac{C}{n-m}+\frac{C_{p}}{n-m} \int_{1}^{t_{\ell}} e^{-\frac{2 V_{\gamma}(r)}{n-m}} \lambda(r)^{2} \mathrm{~d} r+\frac{C_{p}}{n-m} \int_{t_{\ell}}^{t_{\ell+1}} e^{-\frac{2 V_{\gamma}(r)}{n-m}} \lambda(r)^{2} \mathrm{~d} r \\
& \geq \frac{C_{p}}{n-m} \int_{t_{\ell}}^{t_{\ell+1}} e^{-\frac{2 V_{\gamma}(r)}{n-m}} \lambda(r)^{2} \mathrm{~d} r \\
& \geq \frac{C^{2 \ell}}{(n-m)^{2 \ell}} \cdot \frac{(n-m)^{\ell-1}}{C^{\ell-1}}=\left(\frac{C}{n-m}\right)^{\ell+1} .
\end{aligned}
$$


Therefore we prove the claim. In particular, $\lim _{t \rightarrow T-} \lambda(t)=-\infty$ which is the desired contradiction.

Proof of Corollary 2.17. Suppose that there exists a non-negative integrable function $f$ on $\left[0,+\infty\left[\right.\right.$ satisfying $\left\langle V, \nabla r_{p}\right\rangle \geq-f\left(r_{p}\right)$. Then $V_{\gamma}(r) \geq-\int_{0}^{r} f(s) \mathrm{d} s \geq-\int_{0}^{\infty} f(s) \mathrm{d} s>-\infty$ and $\operatorname{Ric}_{m, n}\left(\Delta_{V}\right) \geq 0$ imply

$$
\begin{aligned}
& \int_{0}^{\infty} e^{\frac{2 V_{\gamma}(t)}{n-m}} \operatorname{Ric}_{m, n}\left(\Delta_{V}\right)\left(\dot{\gamma}_{t}, \dot{\gamma}_{t}\right) \mathrm{d} t \\
& \quad \geq \exp \left(-\frac{2}{n-m} \int_{0}^{\infty} f(s) \mathrm{d} s\right) \int_{0}^{\infty} \operatorname{Ric}_{m, n}\left(\Delta_{V}\right)\left(\dot{\gamma}_{t}, \dot{\gamma}_{t}\right) \mathrm{d} t=+\infty
\end{aligned}
$$

This yields the conclusion by Theorem 2.16 .

Proof of Corollary 2.18. Suppose that (2.25) holds for every unit speed geodesic $\gamma$ emanating from $p$. The $(V, m)$-completeness of $(M, g, V)$ at $p$ implies

$$
\int_{0}^{\infty} e^{-\frac{2 V_{\gamma}(t)}{n-m}} \mathrm{~d} t=+\infty
$$

Then we have

$$
\int_{0}^{\infty} e^{\frac{2 V_{\gamma}(t)}{n-m}} \operatorname{Ric}_{m, n}\left(\Delta_{V}\right)\left(\dot{\gamma}_{t}, \dot{\gamma}_{t}\right) \mathrm{d} t \geq(n-m) \kappa C_{p}^{2} \int_{0}^{\infty} e^{-\frac{2 V_{\gamma}(t)}{n-m}} \mathrm{~d} t=+\infty .
$$

This yields the conclusion by Theorem 2.16.

\section{Proof of Theorem 2.20}

For the proof of Theorem 2.20, we need the following lemma on the solution of Jacobi equation.

LEMMA 7.1. Let $\kappa:\left[0, \infty\left[\rightarrow \mathbb{R}\right.\right.$ be a continuous function and $\mathfrak{s}_{\kappa}$ the unique solution of the Jacobi equation $\mathfrak{s}_{\kappa}^{\prime \prime}(s)+\kappa(s) \mathfrak{s}_{\kappa}(s)=0$ with $\mathfrak{s}_{\kappa}(0)=0$ and $\mathfrak{s}_{\kappa}^{\prime}(0)=1$, and $\delta_{\kappa}:=$ $\inf \left\{s>0 \mid \mathfrak{s}_{\kappa}(s)=0\right\}$ the first zero point of $\mathfrak{s}_{\kappa}$. Assume that $\delta_{\kappa}<\infty$ and $\kappa(s)=\kappa\left(\delta_{\kappa}-s\right)$ holds for all $s \in\left[0, \delta_{\kappa}\right]$. Then $\mathfrak{s}_{\kappa}^{\prime}\left(\delta_{\kappa}\right)=-1, \mathfrak{s}_{\kappa}^{\prime}\left(\delta_{\kappa} / 2\right)=0$ and $\mathfrak{s}_{\kappa}(s)=\mathfrak{s}_{\kappa}\left(\delta_{\kappa}-s\right)$ for all $s \in\left[0, \delta_{\kappa}\right]$.

Proof. Set $\overline{\mathfrak{s}}_{\kappa}(s):=\mathfrak{s}_{\kappa}\left(\delta_{\kappa}-s\right)$ for $s \in\left[0, \delta_{\kappa}\right]$. Then this satisfies $\overline{\mathfrak{s}}_{\kappa}^{\prime \prime}(s)+\kappa(s) \overline{\mathfrak{s}}_{\kappa}(s)=0$ and $\overline{\mathfrak{s}}_{\kappa}(0)=0$ and $\overline{\mathfrak{s}}_{\kappa}^{\prime}(0)=-\mathfrak{s}_{\kappa}^{\prime}\left(\delta_{\kappa}\right)$. If we prove $\overline{\mathfrak{s}}_{\kappa}^{\prime}(0)=1$, i.e., $\mathfrak{s}_{\kappa}^{\prime}\left(\delta_{\kappa}\right)=-1$, then the uniqueness of the solution implies the assertion.

Note that $s_{\kappa}(s):=\overline{\mathfrak{s}}_{\kappa}(s) / \overline{\mathfrak{s}}_{\kappa}^{\prime}(0)=-\mathfrak{s}_{\kappa}\left(\delta_{\kappa}-s\right) / \mathfrak{s}_{\kappa}^{\prime}\left(\delta_{\kappa}\right)$ also satisfies the Jacobi equation with $s_{\kappa}(0)=0$ and $s_{\kappa}^{\prime}(0)=1$. Then the uniqueness implies $s_{\kappa}(s)=\mathfrak{s}_{\kappa}(s)$, that is, $\mathfrak{s}_{\kappa}\left(\delta_{\kappa}-s\right)=-\mathfrak{s}_{\kappa}^{\prime}\left(\delta_{\kappa}\right) s_{\kappa}(s)$ for $s \in\left[0, \delta_{\kappa}\right]$, in particular, $\mathfrak{s}_{\kappa}\left(\delta_{\kappa} / 2\right)=-\mathfrak{s}_{\kappa}^{\prime}\left(\delta_{\kappa}\right) \mathfrak{s}_{\kappa}\left(\delta_{\kappa} / 2\right)$. Therefore, $\mathfrak{s}_{\kappa}^{\prime}\left(\delta_{\kappa}\right)=-1$ by $\mathfrak{s}_{\kappa}\left(\delta_{\kappa} / 2\right)>0$. The proof of $\mathfrak{s}_{\kappa}^{\prime}\left(\delta_{\kappa} / 2\right)=0$ is easy from $\mathfrak{s}_{\kappa}^{\prime}(s)=$ $-\mathfrak{s}_{\kappa}^{\prime}\left(\delta_{\kappa}-s\right)$ for $s \in\left[0, \delta_{\kappa}\right]$. 
Hereafter, we assume $V=\nabla \phi$ for some $\phi \in C^{2}(M)$ and set $C_{p}:=\exp \left(-\frac{2 \phi(p)}{n-m}\right)$ for the definition of $s_{p}(x)$ with $p$ being an arbitrary point. We now consider the conformal metric $h=e^{-\frac{4 \phi}{n-m}} g$.

Lemma 7.2. Fix $p \in M$. Suppose that there exists a point $q \in M$ such that $s(p, q)=$ $d^{h}(p, q)$ and let $\gamma$ be the minimal unit speed $g$-geodesic from $p$ and $q$ such that $s(p, q)=$ $\int_{0}^{d(p, q)} e^{-2 \frac{\phi\left(\gamma_{t}\right)}{n-m}} \mathrm{~d}$ t. Then $\nabla \phi$ is parallel to $\dot{\gamma}$ (not parallel along $\left.\gamma\right)$. Moreover if $s(p, x)=$ $d^{h}(p, x)$ holds for any $x \in M$, then $\phi$ is rotationally symmetric around $p$.

Proof. Since $t<d(p, q)$ implies $\gamma_{t} \notin \operatorname{Cut}(p)$, we have $s(p, q)=\int_{0}^{d(p, q)} e^{-2 \frac{\phi\left(\gamma_{t}\right)}{n-m}} \mathrm{~d} t=$ $L^{h}(\gamma)$. Combining this with $s(p, q)=d^{h}(p, q)$ we get $d^{h}(p, q)=L^{h}(\gamma)$. Then $\gamma$ is a minimal geodesic in the $h$ metric. In particular, $\nabla_{\frac{\mathrm{d} \gamma}{\mathrm{d} s}}^{h} \frac{\mathrm{d} \gamma}{\mathrm{d} s}=0$. Applying the formula for connection of $h$ in terms of $g$, we have

$$
\begin{aligned}
0 & =\nabla_{\frac{\mathrm{d} \gamma}{\mathrm{d} s}}^{h} \frac{\mathrm{d} \gamma}{\mathrm{d} s} \\
& =\nabla_{\frac{\mathrm{d} \gamma}{\mathrm{d} s}}^{g} \frac{\mathrm{d} \gamma}{\mathrm{d} s}-\frac{4}{n-m}\left\langle\frac{\mathrm{d} \gamma}{\mathrm{d} s}, \nabla \phi\right\rangle \frac{\mathrm{d} \gamma}{\mathrm{d} s}+\frac{2}{n-m}\left\langle\frac{\mathrm{d} \gamma}{\mathrm{d} s}, \frac{\mathrm{d} \gamma}{\mathrm{d} s}\right\rangle \nabla \phi \\
& =\frac{2 e^{\frac{4 \phi\left(\gamma_{r}\right)}{n-m}}}{n-m}\left(-\left\langle\dot{\gamma}_{r}, \nabla \phi\right\rangle \dot{\gamma}_{r}+\nabla \phi\right) .
\end{aligned}
$$

Then we obtain that $\nabla \phi=\left\langle\nabla \phi, \dot{\gamma}_{r}\right\rangle \dot{\gamma}_{r}$, i.e., $\nabla \phi$ is parallel to $\dot{\gamma}$. Suppose further that $s(p, x)=d^{h}(p, x)$ for any $x \in M$. Let $x_{1}, x_{2} \in M$ be the points in the sphere $\partial B_{r}(p)$ for $r>0$ and $c:[0,1] \rightarrow \partial B_{r}(p)$ a curve on $\partial B_{r}(p)$ joining $c(0)=x_{1}$ and $c(1)=x_{2}$. Then we see $\left\langle\nabla \phi, \dot{c}_{t}\right\rangle=0$, because $\nabla \phi$ is parallel to $\dot{\gamma}$, where $\gamma$ is the $g$-geodesic from $p$ to a point in $\operatorname{Im}(c)$. Hence $\phi\left(x_{2}\right)-\phi\left(x_{1}\right)=\int_{0}^{1}\left\langle\nabla \phi, \dot{c}_{t}\right\rangle \mathrm{d} t=0$.

Here we encounter that $s$ does not necessarily satisfy the triangle inequality. To get around this difficulty we utilize again the conformal metric $h$.

From $d^{h}(p, x) \leq s(p, x)$ and the triangle inequality for the $h$-metric we have

$$
s(p, x)+s(q, x) \geq d^{h}(p, x)+d^{h}(q, x) \geq d^{h}(p, q) .
$$

Proof of Theorem [2.20. First note that $\mathfrak{s}_{\kappa}(s)=\mathfrak{s}_{\kappa}\left(\delta_{\kappa}-s\right)$ holds for $s \in\left[0, \delta_{\kappa}\right]$ by Lemma[7.1. In particular, we have $\cot _{\kappa}(s)=-\cot _{\kappa}\left(\delta_{\kappa}-s\right)$ for all $s \in\left[0, \delta_{\kappa}\right]$. Let $r_{p}$ and $r_{q}$ be the distance functions to $p$ and $q$ respectively. Then by Theorem 2.5 , we have

$$
\Delta_{\nabla \phi}\left(r_{p}+r_{q}\right)(x) \leq(n-m) e^{-\frac{2 \phi(x)}{n-m}}\left(\cot _{\kappa}\left(s_{p}(x)\right)+\cot _{\kappa}\left(s_{q}(x)\right)\right)
$$

holds in the barrier sense. We also have $s_{p}(x)+s_{q}(x) \geq d^{h}(p, q)=\delta_{\kappa}$, so that

$$
\cot _{\kappa}\left(s_{q}(x)\right) \leq \cot _{\kappa}\left(\delta_{\kappa}-s_{p}(x)\right)=-\cot _{\kappa}\left(s_{p}(x)\right) .
$$

Thus, $\Delta_{\nabla \phi}\left(r_{p}+r_{q}\right) \leq 0$ holds in the barrier sense. Note that $\inf _{M}\left(r_{p}+r_{q}\right)$ attains its minimum at a point of minimal geodesic joining $p$ and $q$. Then one can apply the strong minimum principle for superharmonic functions in the barrier sense (see [9, 11] 
for the strong maximum principle for subharmonic functions in the barrier sense) so that $r_{p}(x)+r_{q}(x)=d(p, q)$ for all $x \in M$ and all geodesics starting point at $p$ in $M$ are minimizing and end at $q$. In particular, we have $\Delta_{\nabla \phi}\left(r_{p}+r_{q}\right)=0$ in the classical sense. Therefore, we have

$$
\cot _{\kappa}\left(s_{p}(x)\right)=\cot _{\kappa}\left(\delta_{\kappa}-s_{q}(x)\right) \quad \text { for all } \quad x \in M .
$$

Since $s \mapsto \cot _{\kappa}(s)$ is strictly decreasing, we have $s_{p}(x)+s_{q}(x)=\delta_{\kappa}$. Hence $s_{p}(x)+$ $s_{q}(x)=d^{h}(p, q)=s(p, q)=\delta_{\kappa}$ by $d^{h}(p, q) \leq s(p, q) \leq \delta_{\kappa}$ (see Theorem 2.10). We can apply the similar argument so that $d_{p}^{h}(x)+d_{q}^{h}(x)=d^{h}(p, q)=s(p, q)=\delta_{\kappa}$. Hence $0 \leq s_{p}(x)-d_{p}^{h}(x)=d_{q}^{h}(x)-s_{q}(x) \leq 0$ implies $s_{p}(x)=d_{p}^{h}(x)$. Taking $x \notin \operatorname{Cut}(p)$, we see that there exists a unique minimal unit speed geodesic $\gamma$ with $\gamma_{0}=p$ and $\gamma_{r_{p}(x)}=x$ satisfying $s_{p}(x)=\int_{0}^{r_{p}(x)} e^{-\frac{2 \phi\left(\gamma_{t}\right)}{n-m}} \mathrm{~d} t$. Applying this with Lemma [7.2, $\phi$ is rotationally symmetric around $p$. Secondly, we can deduce that

$$
\begin{aligned}
& \Delta_{\nabla \phi} r_{p}(x)=(n-m) e^{-\frac{2 \phi(x)}{n-m}} \cot _{\kappa}\left(s_{p}(x)\right), \\
& \Delta_{\nabla \phi} r_{q}(x)=(n-m) e^{-\frac{2 \phi(x)}{n-m}} \cot _{\kappa}\left(s_{q}(x)\right)
\end{aligned}
$$

hold in the barrier sense respectively. Consequently, (7.1) (resp. (7.2)) holds for $x \in$ $(\operatorname{Cut}(p) \cup\{p\})^{c}\left(\operatorname{resp} . x \in(\operatorname{Cut}(q) \cup\{q\})^{c}\right)$. Let $\eta$ be a minimal unit speed geodesic from $p$ to $q$ with $\dot{\eta}_{0}=\theta$. Applying Lemma 3.2 to (7.1), we obtain $m=1$ and the expression of a metric of the form

$$
g_{\eta_{r}}=\mathrm{d} r^{2}+e^{\frac{2(\phi(r)+\phi(0))}{n-1}} \mathfrak{s}_{\kappa}^{2}(s(r)) g_{\mathbb{S}^{n-1}}, \quad 0 \leq r \leq d(p, q)
$$

with $s(r)=\int_{0}^{r} e^{-\frac{2 \phi(t)}{n-1}} \mathrm{~d} t$ and $s(d(p, q))=\delta_{\kappa}$. This implies the conclusion.

\section{Proof of Theorem 2.22}

Let $\gamma$ be a ray in $M$, i.e. a unit speed geodesic defined on $\left[0,+\infty\left[\right.\right.$ such that $d\left(\gamma_{t}, \gamma_{s}\right)=$ $|s-t|$ for any $s, t \geq 0$. The Busemann function $b_{\gamma}: M \rightarrow \mathbb{R}$ for a ray $\gamma$ is defined by

$$
b_{\gamma}(x):=\lim _{t \rightarrow+\infty}\left(t-d\left(x, \gamma_{t}\right)\right), \quad x \in M .
$$

It follows from the triangle inequality that $t \mapsto d\left(x, \gamma_{t}\right)$ is monotonically non-decreasing in $t$, so that the above limit exists. Moreover, it is well-known that $b_{\gamma}$ is a 1-Lipschitz function. See e.g. [28].

LEMma 8.1. Let $(M, g)$ be an $n$-dimensional complete Riemannian manifold and $V$ a $C^{1}$-vector field. Fix a point $p \in M$. Suppose that (2.9) holds for any $x \in M$ with $\kappa \equiv 0$. Let $q \in M$ be a point such that $r_{p}$ is smooth at $q$, and let $\gamma$ be the unique unit speed minimal geodesic from $p$ to $q$. Then we have

$$
\left(\Delta_{V} r_{p}\right)(q) \leq \frac{n-m}{\exp \left(\frac{2 V_{\gamma}\left(r_{p}(q)\right)}{n-m}\right) \int_{0}^{r_{p}(q)} \exp \left(-\frac{2 V_{\gamma}(s)}{n-m}\right) \mathrm{d} s} .
$$


Proof. Applying the Riccati inequality (3.2) along $\gamma$ under (2.9) with $\kappa \equiv 0$, we see

$$
\frac{1}{\lambda(r)^{2}} \frac{\mathrm{d} \lambda}{\mathrm{d} r}(r) \leq-\frac{C_{p}}{n-m} e^{-\frac{2 V_{\gamma}(r)}{n-m}}
$$

Integrating this from $\varepsilon>0$ to $r_{p}(q)$ and letting $\varepsilon \rightarrow 0$, we have from $\lim _{\varepsilon \rightarrow 0} \lambda(\varepsilon)=+\infty$ that

$$
\lambda\left(r_{p}(q)\right)=C_{p}^{-1} e^{\frac{2 V_{\gamma}\left(r_{p}(q)\right)}{n-m}}\left(\Delta_{V} r_{p}\right)(q) \leq \frac{n-m}{C_{p} \int_{0}^{r_{p}(q)} e^{-\frac{2 V_{\gamma}(r)}{n-m}} \mathrm{~d} r} .
$$

This implies the conclusion.

Lemma 8.2. Let $(M, g)$ be an n-dimensional complete Riemannian manifold and $V$ a $C^{1}$-vector field. Suppose that $(M, g, V)$ is $(V, m)$-complete. Suppose that (2.11) holds for any $p, x \in M$ with $\kappa=0$. Then the Busemann function $b_{\gamma}$ for any ray $\gamma$ in $M$ is an $\Delta_{V}$-subharmonic function in the barrier sense, i.e., for each $p \in M$ and any $\varepsilon>0$, there exists a smooth function $b_{p, \varepsilon}$ defined on a neighborhood $U_{\varepsilon}(p)$ at $p$ such that $b_{p, \varepsilon}(p)=b_{\gamma}(p)$, $b_{p, \varepsilon} \leq b_{\gamma}$ on $U_{\varepsilon}(p)$, and $\Delta_{V} b_{p, \varepsilon}(p) \geq-\varepsilon$.

Proof. Fix $p \in M$ and a ray $\gamma$ in $M$. Take any sequence $\left\{t_{k}\right\}$ satisfying $\lim _{k \rightarrow \infty} t_{k}=$ $+\infty$. Let $\eta_{t_{k}}$ be a minimal $g$-geodesic joining $p$ and $\gamma_{t_{k}}$. As stated in [11], there exists a subsequence of $t_{k}$ such that the initial vector $\dot{\eta}_{t_{k}}(0)$ converges to some unit vector $u \in T_{p} M$. Let $\eta$ be the ray emanating from $p$ and generated by $u$. Then $p$ does not belong to the cut-locus of $\eta(r)$, hence $\eta(r) \notin \operatorname{Cut}(p)$ for any $r>0$. So $b_{\gamma}^{r}(x):=r-d(x, \eta(r))+b_{\gamma}(p)$ is smooth around $p$ and satisfies $b_{\gamma}^{r} \leq b_{\gamma}$ with $b_{\gamma}^{r}(p)=b_{\gamma}(p)$. By (8.1), we see that for the unique unit speed geodesic $\bar{\gamma}$ from $\eta(r)$ to $p$

$$
\Delta_{V} b_{\gamma}^{r}(p)=-\Delta_{V} r_{\eta(r)}(p) \geq-\frac{n-m}{\exp \left(\frac{2 V_{\bar{\gamma}}(d(\eta(r), p))}{n-m}\right) \int_{0}^{d(\eta(r), p)} \exp \left(-\frac{2 V_{\bar{\gamma}}(t)}{n-m}\right) \mathrm{d} t} .
$$

Note that $\eta(r)=\bar{\gamma}_{d(p, \eta(r))-r}$ for $r \in[0, d(p, \eta(r))]$. Then (8.2) becomes

$$
\Delta_{V} b_{\gamma}^{r}(p)=-\Delta_{V} r_{\eta(r)}(p) \geq-\frac{n-m}{\int_{0}^{d(p, \eta(r))} \exp \left(-\frac{2 V_{\eta}(u)}{n-m}\right) \mathrm{d} u} .
$$

Since $(M, g, V)$ is $(V, m)$-complete, we can construct the desired support function.

Proof of Theorem 2.22. Let $\gamma:]-\infty,+\infty\left[\rightarrow M\right.$ be a line (i.e., $d\left(\gamma_{t}, \gamma_{s}\right)=|s-t|$ for $s, t \in \mathbb{R})$ and $\gamma^{+}, \gamma^{-}$rays defined by $\gamma_{t}^{+}:=\gamma_{t}$ and $\gamma_{t}^{-}:=\gamma_{-t}(t \geq 0)$. Let $b^{+}, b^{-}$ be the Busemann function associated to $\gamma^{+}, \gamma^{-}$, respectively. Then, under the $(V, m)$ completeness of $(M, g, V), b^{+}$and $b^{-}$are continuous $\Delta_{V^{-}}$subharmonic functions on $M$ in the barrier sense by Lemma 8.2 . Since $\gamma$ is a line, for each $x \in M$, we have

$$
b^{+}(x)+b^{-}(x)=\lim _{t \rightarrow+\infty}\left(2 t-d\left(x, \gamma_{t}\right)-d\left(x, \gamma_{-t}\right)\right) \leq 0
$$

and $b^{+}+b^{-}=0$ on $\gamma$. In view of the strong maximum principle for $\Delta_{V}$-subharmonic functions in the barrier sense (see [9, 11] and [12, Lemma 2.4]), we have $b^{+}+b^{-}=0$ on 
$M$. In particular, $b^{+}$and $b^{-}$are continuous $\Delta_{V^{-}}$harmonic functions in the barrier sense. Since $\left|\nabla r_{p}\right|=1$ on $(\operatorname{Cut}(p) \cup\{p\})^{c}$, we have $\left|\nabla b^{+}\right|=\left|\nabla b^{-}\right|=1$ on $M$. Moreover, let $h^{ \pm}$be the smooth $\Delta_{V}$-harmonic function on an open ball $B$ such that $b^{ \pm}=h^{ \pm}$on $\partial B$. Applying the weak maximum principle to the $\Delta_{V}$-harmonic function $b^{ \pm}-h^{ \pm}$on $B$ in the barrier sense, we can deduce $b^{ \pm} \leq h^{ \pm}$on $B$, hence $0=b^{+}+b^{-} \leq h^{+}+h^{-}$. Applying the strong maximum principle again to the smooth $\Delta_{V^{-}}$harmonic function $h^{+}+h^{-}$on $B$, we have $h^{+}+h^{-} \equiv 0$ on $B$. Thus, we can get $0 \geq b^{+}-h^{+}=-\left(b^{-}-h^{-}\right) \geq 0$ on $B$, hence $b^{ \pm}=h^{ \pm}$on $B$. Therefore, $b^{ \pm}$is smooth on any ball $B$, hence on $M$. Applying [34, Lemma 6.5] to the smooth $\Delta_{V^{-}}$-harmonic function $b_{\gamma^{ \pm}}$and $\left|\nabla b_{\gamma^{ \pm}}\right|=1$ on $M$, we can deduce that $\operatorname{Ric}_{1, n}\left(\Delta_{V}\right)\left(\nabla b_{\gamma^{ \pm}}, \nabla b_{\gamma^{ \pm}}\right)=0$ and $n-1$ non-zero eigenvalues of Hess $\left.b^{ \pm}\right|_{p}$ are all equal, because Hess $\left.b^{ \pm}\right|_{p}$ has $n-1$ non-zero eigenvalues. Applying [34, Lemma 6.6] to the smooth $\Delta_{V}$-harmonic function $b^{ \pm}$satisfying $\left|\nabla b^{ \pm}\right|=1$ together with the fact that $\mathrm{CD}(0, m)$-condition implies $\mathrm{CD}(0,1)$-condition for $m<1$, we have that $g$ has a twisted product of the form $g=d r^{2}+e^{\frac{2 \phi}{n-1}} g_{N}$, where $g_{N}$ is a metric on $N$ and $\phi: M \rightarrow \mathbb{R}$ is a smooth function, $\operatorname{Ric}_{1, n}\left(\Delta_{V}\right)\left(\nabla b^{ \pm}, \nabla b^{ \pm}\right)=0$, and $V=\frac{\partial \phi}{\partial r} \cdot \frac{\partial}{\partial r}+U$ with $U \perp \frac{\partial}{\partial r}$. In the same way of the proof of [34, Corollary 6.7], we can deduce that $\frac{d \phi}{d r}=0$, because [34, Proposition 2.1] yields $\operatorname{Ric}_{1, n}\left(\Delta_{V}\right)\left(\frac{\partial}{\partial r}, \frac{\partial}{\partial r}\right)=0$ and

$$
\begin{aligned}
0 \leq \operatorname{Ric}_{m, n}\left(\Delta_{V}\right)\left(\frac{\partial}{\partial r}, \frac{\partial}{\partial r}\right) & =\operatorname{Ric}_{1, n}\left(\Delta_{V}\right)\left(\frac{\partial}{\partial r}, \frac{\partial}{\partial r}\right)+\left(\frac{m-1}{(n-1)(n-m)}\right)\left(\frac{d \phi}{d r}\right)^{2} \\
& =\left(\frac{m-1}{(n-1)(n-m)}\right)\left(\frac{d \phi}{d r}\right)^{2} \leq 0
\end{aligned}
$$

This means that $g$ has the form of product metric $g=d r^{2}+e^{\frac{2 \phi(0, \cdot)}{n-1}} g_{N}=d r^{2}+h_{N}$ on $\mathbb{R} \times N$. Moreover, we can see that $V$ is a vector field on $N$ by using the fact that $\operatorname{Ric}_{m, n}\left(\Delta_{V}\right)\left(\frac{\partial}{\partial r}, U\right)=0$ for all $U \perp \frac{\partial}{\partial r}$.

\section{REFERENCES}

[1] W. Ambrose, A theorem of Myers, Duke Math. J. 24 (1957), no. 3, 345-348.

[2] B. Andrews And L. Ni, Eigenvalue comparison on Bakry-Emery manifolds, Comm. Partial Differential Equations. 37 (2012), no. 11, 2081-2092.

[3] D. BAKRY, L'hypercontractivité et son utilisation en théorie des semigroupes, in: Lecture Notes in Math., vol. 1581, Springer-Verlag, Berlin/New York, 1994, pp. 1-114.

[4] D. Bakry, I. Gentil and M. Ledoux, Analysis and geometry of Markov diffusion operators. Grundlehren der Mathematischen Wissenschaften 348, Springer, Cham, 2014.

[5] D. Bakry and M. Émery, Diffusion hypercontractives, in: Sém. Prob. XIX, in: Lecture Notes in Math., vol. 1123, Springer-Verlag, Berlin/New York, 1985, pp. 177-206.

[6] D. Bakry And M. Ledoux, A logarithmic Sobolev form of the Li-Yau parabolic inequality, Rev. Mat. Iberoam. 22 (2006), no. 2, 683-702.

[7] D. BAKRY AND Z.-M. QIAN, Harnack inequalities on a manifold with positive or negative Ricci curvature, Rev. Mat. Iberoam. 15 (1999), no. 1, 143-179. 
[8] D. BAKRY And Z.-M. Qian, Volume comparison theorems without Jacobi fields, Current trends in potential theory, 115-122, Theta Ser. Adv. Math., 4, Theta, Bucharest, 2005.

[9] E. Calabi, An extension of E. Hopf's maximum principle with an application to Riemannian geometry, Duke Math. J. 25 (1957), no. 1, 45-56.

[10] M. P. Cavalcante, J. Q. Oliveira and M. S. Santos, Compactness in weighted manifolds and applications, Results in Math. 68 (2015), no. 1-2, 143-156.

[11] J.-H. Eschenburg And E. Heintze, An elementary proof of the Cheeger-Gromoll splitting theorem, Ann. Global. Anal. and Geom. 2 (1984), no. 2, 141-151.

[12] F. FAnG, X.-D. Li and Z. Zhang, Two generalizations of Cheeger-Gromoll splitting theorem via Bakry-Emery Ricci curvature, Ann. Inst. Fourier (Grenoble) 59 (2009), no. 2, 563-573.

[13] A. Futaki, H.-Z. Li and X.-D. Li, On the first eigenvalue of the Witten Laplacian and the diameter of compact shrinking Ricci solitons, Ann. Global Anal. Geom. 44 (2013), no. 2, 105-114.

[14] E. P. Hsu, Stochastic analysis on manifolds, Graduate Studies in Mathematics, 38. American Mathematical Society, Providence, RI, 2002.

[15] A. V. Kolesnikov and E. Milman, Poincaré and Brunn-Minkowski inequalities on weighted Riemannian manifolds with boundary, Amer. J. Math. 140 (2018), no. 5, 1147-1185.

[16] K. Kuwae And X.-D. Li, New Laplacian comparison theorem and its applications to diffusion processes on Riemannian manifolds, to appear in Bulletin of London Math. Soc., Available from arXiv:2001.00444

[17] S. Li AND X.-D. LI, W-entropy formula for the Witten Laplacian on manifolds with time dependent metrics and potentials, Pacific J. Math. 278 (2015), no. 1, 173-199.

[18] S. Li And X.-D. Li, Hamilton differential Harnack inequality and W-entropy for Witten Laplacian on Riemannian manifolds, J. Funct. Anal. 274 (2018), no. 11, 3263-3290.

[19] X.-D. Li, Liouville theorems for symmetric diffusion operators on complete Riemannian manifolds, J. Math. Pures Appl. (9) 84 (2005), no. 10, 1295-1361.

[20] X.-D. LI, Perelman's entropy formula for the Witten Laplacian on Riemannian manifolds via BakryEmery Ricci curvature, Math. Ann. 353 (2012), no. 2, 403-437.

[21] J. LotT, Some geometric properties of the Bakry-Émery Ricci tensor, Comment. Math. Helv. 78 (2003), no. 4, 865-883.

[22] J. Lott And C. Villani, Ricci curvature for metric-measure spaces via optimal transport, Ann. of Math. 169 (2009), no. 3, 903-991.

[23] E. Milman, Beyond traditional Curvature-Dimension I: new model spaces for isoperimetric and concentration inequalities in negative dimension, Trans. Amer. Math. Soc. 369 (2017), no. 5, 36053637.

[24] S. Онта, $(K, N)$-convexity and the curvature-dimension condition for negative $N$, J. Geom. Anal. 26 (2016), no. 3, 2067-2096.

[25] S. Ohta And A. TAKatsu, Displacement convexity of generalized relative entropies, Adv. Math. 228 (2011), no. 3, 1742-1787.

[26] P. Petersen, Riemannian geometry, second edition, Graduate Texts in Mathematics, vol. 171, Springer, New York, 2006.

[27] Z.-M. QIAN, Estimates for weight volumes and applications, Quarterly J. Math. 48 (1987), no. 2, $235-242$.

[28] R. Schoen and S. T. Yau, Lectures on differential geometry, Conference Proceedings and Lecture Notes in Geometry and Topology, I. International Press, Cambridge, MA, 1994.

[29] K.-T. Sturm, On the geometry of metric measure spaces. I, Acta Math. 196 (2006), no. 1, 65-131. 
[30] K.-T. Sturm, On the geometry of metric measure spaces. II, Acta Math. 196 (2006), no. 1, 133-177.

[31] H. Tadano, Some Ambrose and Galloway types theorems via Bakry-Émery and modified Ricci curvatures, Pacific J. Math. 294 (2018), no. 1, 213-231.

[32] H. Tadano, Some compactness theorems via $m$-Bakry-Émery and $m$-modified Ricci curvatures with negative $m$, Differential Geometry and its Applications, 75 (2021), 101720.

[33] G. Wei and W. Wylie, Comparison geometry for the Bakry-Emery Ricci tensor, J. Differential Geom. 83 (2009), no. 2, 377-405.

[34] W. Wylie, A warped product version of the Cheeger-Gromoll splitting theorem, Trans. Amer. Math. Soc. 369 (2017), no. 9, 6661-6681.

[35] W. Wylie and D. Yeroshkin, On the geometry of Riemannian manifolds with density, preprint, 2016.

[36] S. Zhu, The Comparison Geometry of Ricci Curvature, Comparison Geometry MSRI Publications 30, (1997), 221-262.

Department of Applied Mathematics

FUKUOKA UNIVERSITY

FUKUOKA 814-0180

JAPAN

Email address: kuwae@fukukoa-u.ac.jp

Oita City Takio Junior High School

ОiтA, 874-0942

JAPAN

Email address: 10.5.hawks61@docomo.ne.jp 